\title{
UNUSUAL PEGMATOID CRYSTALLISATIONS IN A NEPHELINITE PLUG, NEAR ROUND LAGOON, EASTERN CENTRAL PLATEAU, TASMANIA
}

\author{
by F. L. Sutherland, B. J. Barron, R. E. Pogson, J. L. Everard, S. M. Forsyth and J. D. Hollis
}

(with five text-figures, two plates and six tables)

Sutherland, F.L., Barron, B.J., Pogson, R.E., Everard, J.L., Forsyth, S.M. \& Hollis, J.D. 2008 (28:xi): Unusual pegmatoid crystallisations
in a nephelinite plug, near Round Lagoon, eastern Central Plateau, Tasmania. Papers and Proceedings of the Royal Society of
Tasmania 142(2): 23-38. https://doi.org/10.26749/rstpp.142.2.23 ISSN 0080-4703. Geoscience, Australian Museum, 6
College Street, Sydney, New South Wales 2010, Australia (FLS*, REP); 7 Fairview Avenue, St Ives, Sydney, New South Wales
2075, Australia (BJB); Mineral Resources Tasmania, PO Box 56, Rosny Park, Tasmania 7018, Australia (JLE, SMF); "Shenley",
33 Park Street, Trentham, Victoria 3458, Australia (JDH). *Author for correspondence. Email: Lin.Sutherland@austmus.gov.au

Pegmatoids in a Late Oligocene olivine nephelinite plug near Round Lagoon form a complex low-pressure fractionation suite. The host nephelinite contains meta-peridotite and meta-wehrlite mantle xenoliths and its composition ( $\mathrm{Mg \#} \mathbf{0}$.63) may reflect both mantle and then limited low-pressure fractionation. The pegmatoids range from ultramafic through mafic to feldspathic assemblages in a progressive, but discontinuous, fractionation sequence (wehrlite $\rightarrow$ olivine clinopyroxenite $\rightarrow$ sodalite ijolite, ijolite, nepheline syenite $\rightarrow$ alkali syenite). Within this sequence, olivine and clinopyroxene compositions decrease in $\mathrm{Mg}$ content, while clinopyroxene becomes increasingly $\mathrm{Na}$ - and Fe-rich to produce late stage aegirine-augite and aegirine. Nepheline is prominent in the sequence and crystallised over a wide temperature range from $1000^{\circ}$ to $<500^{\circ} \mathrm{C}$. The presence of sodalite suggests volatile Cl-rich fluxing. Mg-rich spinel crystallised in early assemblages, distinct from Fe- and Ti-rich oxides of the magnetite-ulvospinel series in later assemblages. The Round Lagoon low-pressure pegmatoids developed by fractionation in a narrow, vertical feeder rather than in broad lava ponds such as those noted in nephelinite flows at Inverell, New South Wales, and at La Madera, Argentina.

Key Words: olivine nephelinite, fractionation, pegmatoids, feldspathoids, xenoliths, mantle, Tasmania.

\section{INTRODUCTION}

A diverse assemblage of mantle and crustal xenoliths and coarse-grained cognate feldspathoidal and feldspathic crystallisations has been briefly reported from an olivine nephelinite plug, on the eastern Central Plateau in Tasmania (Sutherland \& Hollis 1982, Sutherland 1989, Sutherland et al. 1989, Everard 2001). The site lies $0.7 \mathrm{~km}$ east of Round Lagoon and $7 \mathrm{~km}$ north of Lake Sorell (Grid AMG $517600 \mathrm{mE}, 5348400 \mathrm{mN}$, or $147^{\circ} 13.7^{\prime} \mathrm{E}, 42^{\circ} 00.9^{\prime} \mathrm{S}$, datum AGD 66). The plug is a prominent, strongly east-west-jointed feature about $250 \mathrm{~m}$ across, which penetrates the eroded edge of the Jurassic dolerite sill, here about $300 \mathrm{~m}$ thick, which forms the Great Western Tiers. It extends from 960-1030 $\mathrm{m}$ asl, with its greatest exposure on the down slope side. Brecciated and altered dolerite is visible against the north side of the plug, suggesting intrusive emplacement of the nephelinite, but scree obscures the contact elsewhere. This olivine nephelinite plug was analysed and dated by the K-Ar

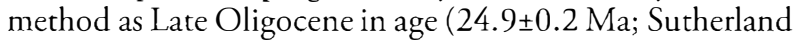
1989). It is one of many basaltic plug and fow remnants mapped on the Interlaken 1:50000 geological map sheet (Forsyth 1989). These lie west of the Tiers escarpment, formed by erosional retreat inland from the Tiers lineament that bounds the Midlands rift valley to the east (Direen \& Leaman 1997). The lithospheric mantle below this region lies at a depth of about $30 \mathrm{~km}$ (Rawlinson et al. 2001).

The xenolithic and coarse cognate assemblages here form one of the most varied, and best preserved, inclusion suites in Tasmania. As well as containing fragments from the Jurassic dolerite and underlying Permian sandstones and shales, ultramafic mantle xenoliths, some cumulatetextured ultramafic to mafic pyroxenites, disaggregated xenocrysts, and coarse pegmatoidal feldspathoidal and feldspathic crystallisations also occur. The mantle xenoliths are dominated by spinel meta-lherzolite, mostly mediumgrained, but finer grained types and recrystallised, partly glassy variants occur. Some olivine grains within them, up to $50 \mathrm{~mm}$ across, approach gem quality, but are mostly fractured. Some coarse spinel meta-lherzolites and pyroxenites exhibit lamellar exsolution of clinopyroxene in orthopyroxene. Mantle xenoliths reach up to $210 \mathrm{~mm}$ across. One has a composite structure (fig. 1), in which spinel meta-lherzolite is disrupted by cumulate crystallisations of transitional wehrlite $(-40 \%$ olivine, $60 \%$ clinopyroxene) showing a cavity-like texture which have finer grained margins of clinopyroxenite ( - olivine $5 \%$, clinopyroxene 95\%) against the host meta-lherzolite.

Abundant coarse-grained pegmatoids in the host nephelinite which have feldspathoidal and syenitic assemblages are the focus of the current investigation. One long pegmatoid vein traverses the plug, but most pegmatoids

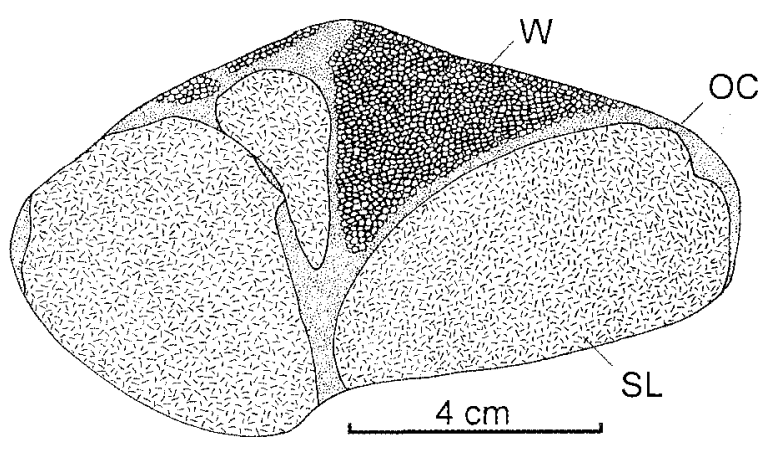

FIG. 1 - Composite mantle xenolith, showing spinel lherzolite $(S L)$, intruded by finer grained margins of cumulate olivine clinopyroxenite (OC) and coarse cumulate wehrlite (W). Sketch by J.D. Hollis, redrawn by R.T. Springthorpe. 
form discrete, subangular bodies up to a few cm across. Some rounded bodies contain internal radial growths and represent cavity fillings. Most, however, show abrupt, irregular contacts with the host. Such abundant feldspathoidal pegmatoids are rarely described from olivine nephelinites (London 2008). A single nepheline-sodalite-syenite pegmatoid from Boat Harbour, NW Tasmania (Sutherland et al. 1996), forms an isolated example. Pegmatoidal developments in nephelinite near Inverell, New South Wales (Wilkinson 1977), are not as mineralogically diverse as those at Round Lagoon. Abundant pegmatitic dykes occur in melanephelinite from Córdoba, Argentina (Galliski et al. 2004), but these are injections into a flow and differ in mineralogy to Round Lagoon pegmatoids. Pegmatoids and their low-pressure fractionation trends within olivine-melilite-nephelinite flows in Hawaii (Wilkinson \& Stolz 1983) and western Bohemia (Ulrych et al. 2000) differ in that their assemblages include melilite. Alkali pyroxenite and ijolite materials in a nephelinite centre from Bohemia that were originally thought to be endogenous are now considered to represent a separate, deep-seated nephelinitic crystallisation (Ulrych et al. 2005).

This present paper documents the unusual Round Lagoon xenolith and pegmatoidal mineral assemblages, particularly the late-stage assemblages. The results are then used to evaluate the pegmatoidal assemblages in terms of fractionation trends described from nephelinites elsewhere and also compare the high-pressure ultramafic xenoliths with other Tasmanian and eastern Australian mantle suites. The term pegmatoid is preferred here over the term pegmatite, which includes much coarser assemblages associated with larger intrusive masses of alkaline and silicic rocks (London 2008).

\section{MATERIALS AND ANALYTICAL METHODS}

Thin sections and polished thin sections were prepared from samples housed in the Australian Museum collections. The study suite with sample numbers is listed in table 1. Two electron microprobe (EMP) facilities were used for mineral analyses. Analyses made on automated ETEC microprobe at Macquarie University, North Ryde, used natural standards, Bence-Albee matrix corrections, a $15 \mathrm{kV}$ accelerating voltage and a sample current of $20 \mathrm{nA}$ (B.J. Barron, analyst). Supplementary analyses under similar settings employed a combined wave length energy dispersive system at the Electron Microscope Unit, University of Sydney, with wave length spectrometers used to improve detection limits on key elements such as $\mathrm{Na}$ and $\mathrm{K}$ (D.F. Hendry, analyst). These methods gave precisions better than \pm 1 relative $\%$ for elements above $10 \mathrm{wt} \%$ as oxides, $\pm 5 \%$ for elements between $1-10 \mathrm{wt} . \%$ as oxides and $\pm 10 \%$ for elements below $1 \mathrm{wt} . \%$

TABLE 1

Samples studied from Round Lagoon nephelinite plug

\begin{tabular}{|c|c|c|c|c|}
\hline Sample & Nature & Examination 1 & Designation $^{2}$ & Origin \\
\hline RL1 & Peridotite & TS, OM, PM & Spl-wehrlite & Cumulate \\
\hline \multirow[t]{2}{*}{ RL1A } & Pyroxenite & TS, OM, PM & Ol-ne-clinopyroxenite & Cumulate \\
\hline & Foid-syenitoid & & Ijolite $^{3,4}$ & Late-stage \\
\hline RL2 & Peridotite & TS,OM & Spl-wehrlite & Cumulate \\
\hline RL2A & Foid-syenitoid & TS,OM, EMP, PM & Ijolite ${ }^{3}$ & Late-stage \\
\hline RL3 & Foidolite & TS,OM, EMP, PM & Sdl-ijolite 4 & Late-stage \\
\hline RL3A & Nephelinite host & TS,OM, EMP & Ol-nephelinite 3 & Eruptive \\
\hline RL3B & Pyroxenite & TS,OM & Ol-ne-clinopyroxenite & Cumulate \\
\hline RL4 & Foidolite & $\mathrm{TS}, \mathrm{OM}$ & Ijolite $^{3}$ & Late-stage \\
\hline RL5 & Pyroxenite & $\mathrm{TS}, \mathrm{OM}$ & Ol-ne-clinopyroxenite & Cumulate \\
\hline RL6 & Foid-syenitoid & TS,OM, EMP, PM & Ne-syenite ${ }^{3}$ & Late-stage \\
\hline RL6A & Peridotite & TS,OM, EMP, PM & Spl-wherlite & Meta-mantle \\
\hline RL7 & Pyroxenite & $\mathrm{TS}, \mathrm{OM}$ & Spl-ol-clinopyroxenite & Meta-mantle \\
\hline RL7A & Foid-syenitoid & $\mathrm{TS}, \mathrm{OM}$ & Ne-syenite 3 & Late-stage \\
\hline RL8 & Peridotite & TS,OM, EMP, PM & Spl-wehrlite ${ }^{4}$ & Cumulate \\
\hline RL9 & Peridotite & TS,OM, EMP, PM & Spl-lherzolite & Meta-mantle \\
\hline RL10 & Pyroxenite & $\mathrm{TS}, \mathrm{OM}$ & Ol-clinopyroxenite & Cumulate \\
\hline RL1 1 & Foid-syenitoid & $\mathrm{TS}, \mathrm{OM}$ & Ne-syenite & Late-stage \\
\hline RL12 & Foid-syenitoid & $\mathrm{TS}, \mathrm{OM}$ & Ne-syenite & Late-stage \\
\hline RL13 & Pyroxenite & $\mathrm{TS}, \mathrm{OM}$ & Spl-clinopyroxenite & Meta-mantle \\
\hline RL 14 & Pyroxenite & $\mathrm{TS}, \mathrm{OM}$ & Spl-ol-websterite & Meta-mantle \\
\hline RL15 & Microsyenitoid & TS,OM, EMP, PM & Alkali syenite 3,4 & Late-stage \\
\hline RL16 & Pyroxenite & $\mathrm{TS}, \mathrm{OM}$ & Spl-websterite & Meta-mantle \\
\hline RL17 & Peridotite & $\mathrm{TS}, \mathrm{OM}$ & Olivine megacryst & Meta-mantle \\
\hline RL.18 & Peridotite & $\mathrm{TS}, \mathrm{OM}$ & Spl-lherzolite & Meta-mantle \\
\hline RL19 & Pyroxenite & TS,OM, EMP, PM & Ol-clinopyroxenite & Cumulate \\
\hline
\end{tabular}

${ }^{1}$ TS, thin/polished section; OM, optical microscopy; EMP, electron microprobe; PM, photomicrograph.

${ }^{2}$ Spl, spinel. Ol, olivine. Ne, nepheline. Sdl, sodalite.

${ }^{3} \mathrm{Afd}$, alkali feldspar. ${ }^{4}$ Amp, amphibole. 
as oxides. Comparative analyses from the two instruments mostly agreed within $\pm 2 \%$ for oxides above $5 \mathrm{wt} . \%$, within $\pm 8 \%$ for oxides between $1-4$ wt. $\%$ and $\pm 30 \%$ for oxides below 1 wt.\%. A semi-quantitative Energy Dispersive X-ray Spectrometer (Oxford Instruments Link Isis 200) at the Australian Museum, Sydney (R.E. Pogson, analyst), was used to determine the $\mathrm{Cl}$ content of sodalite. Photomicrographs of sections were taken using a Leica DMLP petrographic microscope, with a digital image capture systems, at the Australian Museum (pls 1, 2).

Representative analyses selected from the wider EMP data set are listed for the essential petrological assemblages in tables $2-5$. The geochemical data were plotted on triangular plots and discrimination diagrams, using Geo Plot (Zhou \& Li 2006). Mineral nomenclature follows International Mineralogical Association guidelines. End members for olivine are based on Deer et al. (1982). Pyroxene names are based on Morimoto (1988) and the computer program of Cebeira (1990). Amphibole names are based on Leake et al. (1997) and the computer program of Yavuz (1996). Rock names follow general recommendations in Le Maitre (2002) and for Australian intraplate volcanic rocks those in Johnson (1989). Normative mineral abbreviations follow those in Hutchison (1974), while symbols for rock-forming minerals follow Kretz (1983).

\section{PETROGRAPHIC RESULTS}

\section{Host nephelinite}

The nephelinite contains olivine and clinopyroxene microphenocrysts up to $2 \mathrm{~mm}$ across in a groundmass of granular olivine, prismatic clinopyroxene, granular to subhedral equant opaque oxide grains and interstitial and partly sub-ophitic to poikilitic nepheline and alkali feldspar. Acicular apatite and small ragged flakes of dark mica are accessories. Mineral analyses (table 2 ) show the olivine is forsterite $\left(\mathrm{fo}_{72-74}\right)$, partly corroded and altered to "iddingsite". Clinopyroxene is zoned titaniferous augite-diopside $\left(\mathrm{en}_{41-49} \mathrm{di}_{40-49} \mathrm{fs}_{10-11} ; \mathrm{TiO}_{2}\right.$ $2-3 \mathrm{wt} . \%$ ) with simple to multiple twinning and partially resorbed cores in phenocrysts. Opaque oxide is magnetiteulvospinel, which forms sporadic clusters. Nepheline is sodic $\left(\mathrm{ne}_{80-81} \mathrm{kls}_{14-15} \mathrm{qtz}_{2-3}\right.$ ); alkali feldspar is zoned from anorthoclase to sanidine $\left(\mathrm{or}_{52} \mathrm{ab}_{47} \mathrm{an}_{1}\right)$. Although overlapping, the crystallisation sequence is: olivine, clinopyroxene, ulvospinel, apatite, nepheline, alkali feldspar and zeolites.

\section{Ultramafic cumulates}

These comprise wehrlites and olivine-clinopyroxenites. The wehrlites (RL1, 2, 8; table 2 ; pl. 2A) contain up to $85 \%$ olivine $\left(\mathrm{fo}_{90-91} \mathrm{fa}_{9-10}\right)$ as coarse, granular grains up to $5 \mathrm{~mm}$ across and as smaller inclusions in branching domains of microgranular pale green Cr-bearing augite $\left(\mathrm{en}_{54-55} \mathrm{wo}_{40-42} \mathrm{fs}_{4}\right.$; $\mathrm{Cr}_{2} \mathrm{O}_{3} 2.2-2.5$ wt.\%). Accessory interstitial yellow-brown Cr-bearing spinel ( $\mathrm{spl}_{82-83} \mathrm{Chr}_{13-14} \mathrm{usp}_{2} \mathrm{mag}_{1-2} \mathrm{hc}_{1}$ ) exhibits opaque reaction rims. Residual, branching networks and veinlets through the assemblage are composed of glassy, low relief material that contains rare phlogopite grains $(\mathrm{Mg}$ \# 0.87 ), alkali feldspar, saponitic clays and other alteration products.

The olivine-clinopyroxenites (RL1A, 3B, 5, 10, 19; table 2; pl. 2B) contain intergrown crystals of $\mathrm{Ti}$ - and $\mathrm{Al}$ - bearing diopside ( $\mathrm{wo}_{51-57} \mathrm{en}_{37-38} \mathrm{fs}_{10-12} ; \mathrm{Al}_{2} \mathrm{O}_{3} 5.2-5.6 \mathrm{wt} . \%, \mathrm{TiO}_{2}$

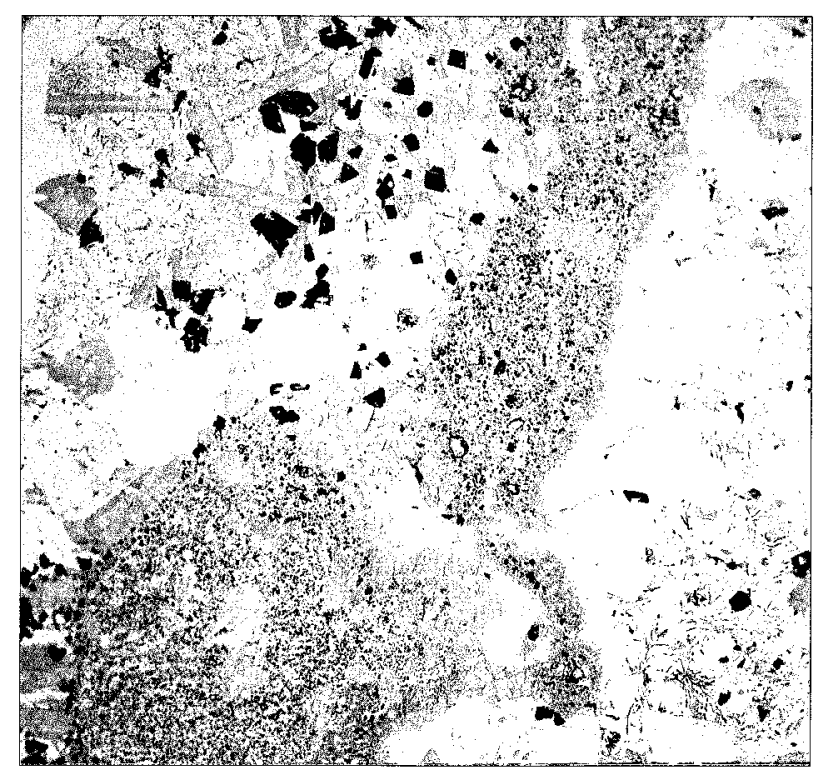

\section{PLATE 1}

Photomicrograph of olivine clinopyroxenite (bottom left) and surrounding sodalite-ijolite (right) and ijolite (top left) crystallisations in host olivine nephelinite (centre). Field of view $23 \mathrm{~mm}$ wide $X 22 \mathrm{~mm}$ high. R.E. Pogson photograph.

2.3-2.8 wt.\%), commonly up to $8 \mathrm{~mm}$ across or more in some cases. They include small grains of olivine $\left(\mathrm{fo}_{73}\right)$. Interstitial and vein-like intergrowths consist of sodic nepheline ( $\mathrm{ne}_{76-77} \mathrm{kls}_{13-15} \mathrm{qtz}_{10}$ ) and accessory alkali feldspar, ulvospinel, apatite and rare brown mica. These may form along zones of alteration in diopside. In contact with the host nephelinite, the diopside has euhedral overgrowths which lack inclusions. Rare, composite diopside aggregates show crystal growth radiating out from a nucleus of sodalite, alkali feldspar and poikilitic nepheline that encloses small olivine grains.

A notable composite pegmatoid in the host nephelinite (RL1A) contains a nepheline-olivine-bearing clinopyroxenite core within flanking ijolite and nepheline syenite pegmatoid. This illustrates a localised fractionation process within crystallising nephelinite (pl. 1).

\section{Feldspathoid-rich pegmatoids}

These foidolites range from sodalite- and nepheline-rich assemblages to nepheline-rich assemblages containing minor alkali feldspar. Typical sodalite- $\left(\mathrm{sdl}_{93} \mathrm{k}-\mathrm{sdl}_{7}\right)$ and nepheline (ne ${ }_{67-68} \mathrm{kls}_{29-30} \mathrm{qtz}_{3}$ )-rich examples (RL3, table 3, pl. 2C) contain about $50 \%$ interstitial feldspathoids (nepheline slightly exceeds sodalite) and $50 \%$ stumpy subhedral to euhedral pale yellow diopside $\left(w_{46-49} \mathrm{en}_{33-43} \mathrm{fs}_{9-19}\right)$, partially grading to pinkish Ti-bearing $\left(\mathrm{TiO}_{2} 2\right.$ wt.\%) and dark blue-green sodic pyroxene on its rims and in adjacent grains $\left(\operatorname{acm}_{53-57} \mathrm{wo}_{17-21} \mathrm{en}_{10-15} \mathrm{fs}_{8-11} \mathrm{jd}_{2}\right)$. The diopside contains small inclusions of olivine $\left(\mathrm{fo}_{65-66}\right)$, accessory ulvospinel and aegirine-augite.

Nepheline- and alkali feldspar-rich assemblages (RL $1 \mathrm{~A}, 2 \mathrm{~A}, 4,6,11,12$; table 3 , pl. 2E) contain nepheline $\left(\mathrm{ne}_{82-83} \mathrm{kls}_{15-16} \mathrm{qtz}_{2}\right)$ as oriented narrow hexagonal prismatic crystals in coarser anhedral anorthoclase $\left(\mathrm{ab}_{59-61} \mathrm{Or}_{33-35} \mathrm{an}_{6}\right)$ and associated domain-zoned, anhedral pale purple $\mathrm{Ti}$ - and Al-bearing diopside $\left(\mathrm{wo}_{50-52} \mathrm{en}_{36-41} \mathrm{fs}_{9-13} ; \mathrm{TiO}_{2} 2-4 \mathrm{wt} \%\right.$, 

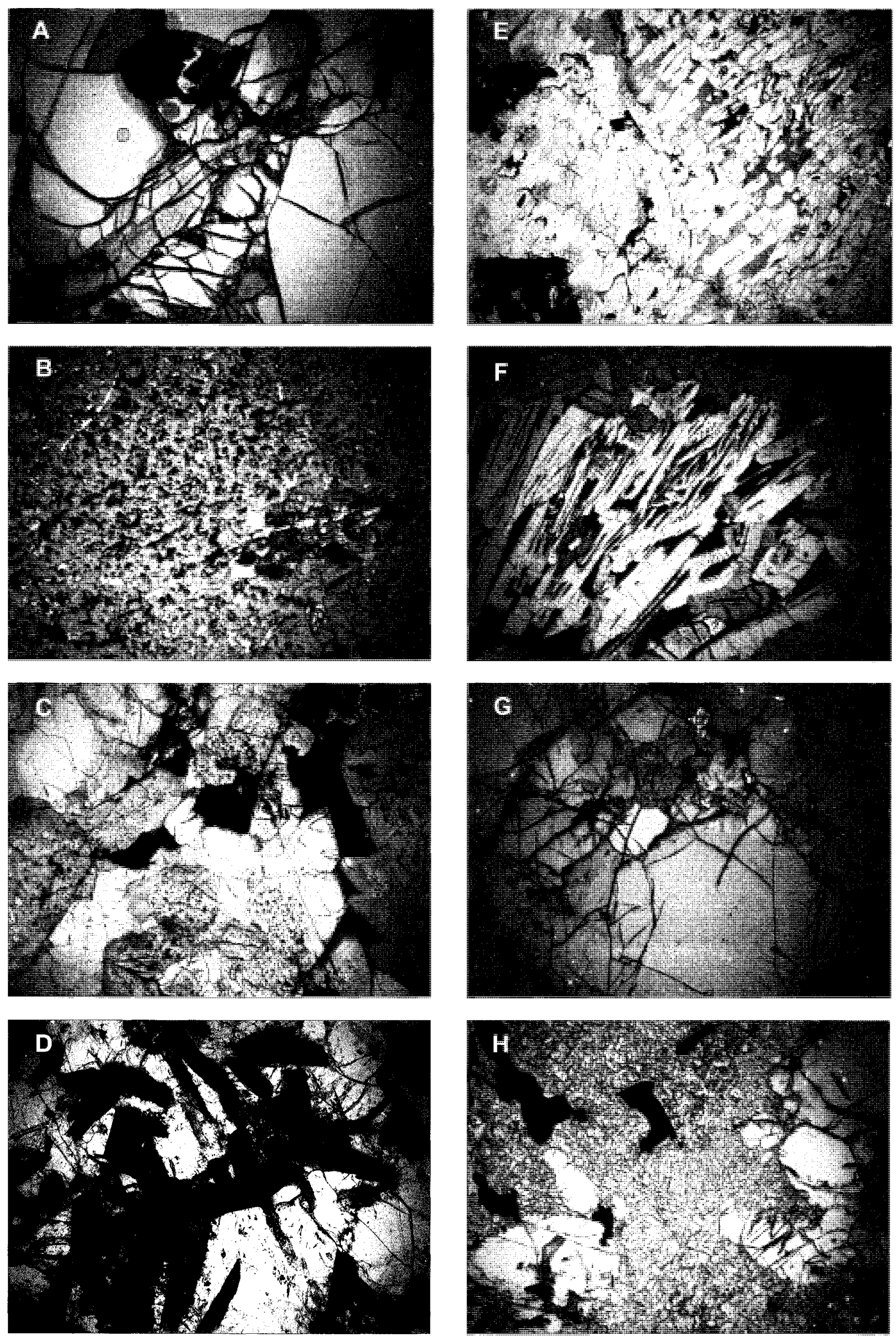
TABLE 2

Representative analyses host nephelinite and ultramafic cumulates

\begin{tabular}{|c|c|c|c|c|c|c|c|c|c|c|}
\hline \multirow{2}{*}{$\begin{array}{l}\text { Analysis wt. } \% \\
\text { Mineral }\end{array}$} & \multicolumn{3}{|c|}{$\begin{array}{l}\text { Ol-nephelinite } \\
\quad(\text { RL3A })^{1}\end{array}$} & \multicolumn{4}{|c|}{$\begin{array}{l}\text { Wehrlite } \\
(\text { RL8 })^{2}\end{array}$} & \multicolumn{3}{|c|}{$\begin{array}{c}\text { Olv-clinopyroxenite } \\
(\text { RL19) })^{3} \\
\end{array}$} \\
\hline & $\mathrm{Ol}$ & $\mathrm{Cpx}$ & $\mathrm{Ne}$ & $\mathrm{Ol}$ & $\mathrm{Cpx}$ & $S_{\mathrm{pl}}$ & Clay & $\mathrm{Cpx}$ & $\mathrm{OI}$ & $\mathrm{Ne}$ \\
\hline $\mathrm{SiO}_{2}$ & 38.62 & 48.95 & 44.33 & 40.82 & 54.88 & 0.59 & 38.12 & 49.03 & 38.35 & 47.48 \\
\hline $\mathrm{TiO}_{2}$ & & 2.42 & 0.16 & & & 0.14 & & 2.33 & 0.04 & 0.06 \\
\hline $\mathrm{Al}_{2} \mathrm{O}_{3}$ & & 5.14 & 32.64 & & & 53.69 & 15.19 & 5.34 & & 31.67 \\
\hline $\mathrm{Cr}_{2} \mathrm{O}_{3}$ & & 0.24 & & & 2.55 & 13.51 & 0.10 & 0.29 & & \\
\hline $\mathrm{FeO}$ & 20.39 & 6.04 & 0.85 & 8.74 & 2.66 & 9.18 & 0.29 & 4.98 & 24.32 & 0.76 \\
\hline $\mathrm{Fe}_{2} \mathrm{O}_{3}$ & & & & & & 1.69 & & 1.20 & & \\
\hline $\mathrm{MnO}$ & 0.27 & & & & 0.17 & & 0.11 & 0.12 & 0.77 & 0.09 \\
\hline $\mathrm{MgO}$ & 40.14 & 13.69 & & 49.57 & 18.39 & 21.01 & 25.88 & 12.67 & 36.62 & 0.26 \\
\hline $\mathrm{CaO}$ & 0.32 & 22.07 & 0.88 & 0.13 & 18.73 & & 0.71 & 24.18 & 0.26 & 0.32 \\
\hline $\mathrm{Na}_{2} \mathrm{O}$ & & 0.68 & 16.48 & & 1.26 & & & 0.41 & & 15.32 \\
\hline $\mathrm{K}_{2} \mathrm{O}$ & & & 4.15 & & & & & 0.05 & & 3.86 \\
\hline Total & 99.74 & 99.23 & 99.49 & 99.26 & 99.11 & 99.81 & 80.40 & 100.50 & 100.36 & 99.82 \\
\hline Cations (No. O) & 4 & 6 & 32 & 4 & 6 & 32 & 20 & 6 & 4 & 32 \\
\hline $\mathrm{Si}^{4+}$ & 0.999 & 1.826 & 8.500 & 1.002 & 1.998 & 0.124 & 5.336 & 1.814 & 1.005 & 8.945 \\
\hline $\mathrm{Ti}^{4+}$ & & 0.068 & 0.023 & & & 0.021 & & 0.065 & 0.001 & 0.009 \\
\hline $\mathrm{Al}^{3+}$ & & 0.226 & 7.379 & & & 13.215 & 2.506 & 0.233 & & 7.035 \\
\hline $\mathrm{Cr}^{3+}$ & & 0.007 & & & 0.073 & 2.231 & 0.011 & 0.009 & & \\
\hline $\mathrm{Fe}^{2+}$ & 0.441 & 0.152 & 0.136 & 0.179 & 0.081 & 1.603 & 0.034 & 0.154 & 0.533 & 0.120 \\
\hline $\mathrm{Fe}^{3+}$ & & 0.036 & & & & 0.2648 & & 0.033 & & \\
\hline $\mathrm{Mn}^{2+}$ & 0.006 & & & & 0.005 & & 0.013 & 0.004 & 1.431 & 0.014 \\
\hline $\mathrm{Mg}^{2+}$ & 1.547 & 0.761 & & 1.813 & 1.023 & & 5.399 & 0.699 & 0.007 & 0.073 \\
\hline $\mathrm{Ca}^{2+}$ & 0.009 & 0.882 & 0.180 & 0.003 & 0.730 & 6.566 & 0.106 & 0.958 & & 0.065 \\
\hline $\mathrm{Na}^{1+}$ & & 0.049 & 6.126 & & 0.089 & & & 0.029 & & 5.598 \\
\hline $\mathrm{K}^{1+}$ & & & 1.015 & & & & & 0.002 & & 0.928 \\
\hline Sum & 3.001 & 4.007 & 23.358 & 2.998 & 4.001 & 24.098 & 12.405 & 4.000 & 2.994 & 22.790 \\
\hline \multicolumn{11}{|l|}{ Cation ratio $(\%)$} \\
\hline $\mathrm{Mg}$ & 77.5 & 41.5 & & 90.8 & 55.8 & 61.3 & 97.5 & 37.8 & 73.0 & \\
\hline $\mathrm{Fe}$ & 22.1 & 10.3 & & 9.0 & 4.4 & 17.7 & 0.6 & 10.3 & 27.0 & \\
\hline $\mathrm{Cr}$ & & & & & & 21.0 & & & & \\
\hline $\mathrm{Ca}$ & 0.4 & 48.2 & 2.46 & 0.2 & 39.8 & & 1.9 & 51.9 & & 0.9 \\
\hline $\mathrm{Na}$ & & & 83.7 & & & & & & & 85.0 \\
\hline $\mathrm{K}$ & & & 13.9 & & & & & & & 14.1 \\
\hline
\end{tabular}

${ }^{1}$ RL3A Ol: Forsterite $\left(\mathrm{fo}_{78} \mathrm{fa}_{22}\right)$; Cpx: Diopside $\left(\mathrm{wo}_{48} \mathrm{en}_{42} \mathrm{fs}_{10}\right)$; Ne: Nepheline $\left(\mathrm{ne}_{80} \mathrm{kls}_{14} \mathrm{qtz}\right.$ ).

2 RL8 Ol: Forsterite ( $\left.\mathrm{fo}_{91} \mathrm{fa}_{9}\right)$; Cpx: Augite $\left(\mathrm{en}_{55} \mathrm{wo}_{40} \mathrm{fs}_{5}\right)$; Spl: Cr-rich spinel ( $\left.\mathrm{sp}_{82} \mathrm{chr}_{14} \mathrm{usp}_{2} \mathrm{mag}_{2} \mathrm{hc}_{1}\right)$;

${ }^{3}$ RL19 Cpx: Diopside ( $w_{52} \mathrm{en}_{38} \mathrm{fs}_{10}$ ); Ol: Fosterite ( $\left.\mathrm{fo}_{73} \mathrm{fa}_{27}\right)$; Ne: Nepheline (ne $\left.\mathrm{e}_{77} \mathrm{kls} \mathrm{s}_{13} \mathrm{qtz} \mathrm{z}_{10}\right)$.

PLATE 2 (opposite page)

Photomicrographs of selected pegmatoid and xenolith textures within the Round Lagoon nephelinite. Field of view for each image, $5 \mathrm{~mm}$ wide X $3.5 \mathrm{~mm}$ high. A. Wehrlite (RL8), with olivine (large grains), Cr-rich augite (long crystals, centre and bottom left) and Cr-bearing spinel (opaque grain, centre top). B. Olivine-nepheline-clinopyroxenite (RL 19) with diopside (large grains) riddled with olivine (small crystals, light and dark altered grains) and nepheline (light coloured crystals). C. Sodalite ijolite (RL3) with nepheline and sodalite (light crystals), diopside (spongy included cores), Ti-rich diopside (rims), aegirine-augite (dark overgrowths, bottom right) and ulvospinel (opaque interstitial crystals, top). D. Ijolite (RL2A), with nepheline and anorthoclase (light crystals), strongly zoned Ti-rich diopside (dark crystals). E. Nepheline syenite (RL6), with nepheline (light aligned crystals), sanidine (light coloured enclosure, centre left), diopside (intergrown darker area) and ulvospinel (large crystals, bottom left). F. Alkali syenite (RL15) with sanidine (light aligned crystals), diopside (small crystals top centre) and aegirine-augite and aegirine (dark intergrowth). G. Spinel meta-lherzolite (RL9) with olivine (large crystal, centre bottom), diopside (crystal centre top) and orthopyroxene (centre right and top left). H. Altered meta-wehrlite (RL6A) with olivine (crystals top right and bottom left), diopside (crystals bottom right), Cr-bearing spinel (black irregular grains) and extensive fune grained recrystallised zones. All images taken in plane polarised light. R.E. Pogson photographer. 
TABLE 3

Representative mineral analyses, ijolitic pegmatoids

\begin{tabular}{|c|c|c|c|c|c|c|c|c|c|c|}
\hline \multirow{2}{*}{$\begin{array}{l}\text { Analysis wt. \% } \\
\text { Mineral }\end{array}$} & \multicolumn{5}{|c|}{ Sodalite ijolite (RL3) } & \multicolumn{5}{|c|}{ Ijolite (RL 2A) } \\
\hline & $\mathrm{Ne}$ & Sdl & $\mathrm{Cpx}^{1}$ & $\mathrm{Cpx}^{2}$ & $\mathrm{Ol}$ & Nep & Afd & $C_{p x}$ & $\mathrm{Ol}$ & Spl \\
\hline $\mathrm{SiO}_{2}$ & 42.10 & 38.19 & 54.73 & 51.60 & 37.20 & 42.18 & 62.73 & 47.47 & 37.42 & 0.65 \\
\hline $\mathrm{TiO}_{2}^{2}$ & & & 0.20 & 3.97 & & & 0.44 & 3.42 & 0.02 & 21.26 \\
\hline $\mathrm{Al}_{2} \mathrm{O}_{3}$ & 33.19 & 30.89 & 0.22 & 0.35 & 0.03 & 34.26 & 20.71 & 4.95 & 0.05 & 0.60 \\
\hline $\mathrm{Cr}_{2} \mathrm{O}_{3}$ & & & 0.12 & & & & & 0.09 & 0.05 & 0.77 \\
\hline $\mathrm{FeO}$ & 1.04 & 0.72 & 5.98 & 16.67 & 28.56 & 0.86 & 0.38 & 7.36 & 21.70 & 46.38 \\
\hline $\mathrm{Fe}_{2} \mathrm{O}_{3}$ & & & & 7.12 & & & & & & 27.39 \\
\hline $\mathrm{MnO}$ & & 0.01 & 0.12 & 0.09 & 0.85 & 0.03 & 0.01 & 0.20 & 0.54 & 0.61 \\
\hline $\mathrm{MgO}$ & 0.39 & & 14.89 & 3.24 & 31.67 & 0.17 & 0.01 & 12.50 & 39.15 & 2.74 \\
\hline $\mathrm{CaO}$ & 0.06 & 0.10 & 24.04 & 7.19 & 0.74 & 1.64 & 1.29 & 23.55 & 0.52 & 0.79 \\
\hline $\mathrm{Na}_{2} \mathrm{O}$ & 14.00 & 21.89 & 0.14 & 9.29 & & 16.40 & 6.62 & 0.46 & & \\
\hline $\mathrm{K}_{2} \mathrm{O}$ & 8.72 & 2.39 & 0.09 & & & 4.50 & 5.87 & 0.02 & & \\
\hline $\mathrm{Cl}$ & & 7.37 & & & & & & & & \\
\hline $\mathrm{O} \equiv \mathrm{Cl}$ & & -1.66 & & & & & & & & \\
\hline Total & 99.50 & 99.89 & 100.53 & 99.49 & 99.05 & 100.04 & 98.06 & 100.29 & 99.45 & 101.19 \\
\hline Cations (No. O) & 32 & $26(+\mathrm{Cl})$ & 6 & 6 & 4 & 32 & 32 & 6 & 4 & 32 \\
\hline $\mathrm{Si}^{4+}$ & 8.239 & 6.179 & 2.008 & 1.982 & 1.012 & 8.109 & 11.496 & 1.774 & 0.981 & 0.188 \\
\hline $\mathrm{Ti}^{4+}$ & & & 0.006 & 0.115 & & & 0.061 & 0.096 & 0.000 & 4.634 \\
\hline $\mathrm{Al}^{3+}$ & 7.655 & 5.890 & 0.010 & 0.016 & 0.001 & 7.763 & 4.473 & 0.218 & 0.002 & 0.205 \\
\hline $\mathrm{Cr}^{3+}$ & & & & & & & & 0.003 & 0.001 & 0.177 \\
\hline $\mathrm{Fe}^{2+}$ & 0.17 & 0.097 & 0.184 & 0.229 & 0.650 & 0.138 & 0.058 & 0.156 & 0.476 & 11.243 \\
\hline $\mathrm{Fe}^{3+}$ & & & & 0.482 & & & & 0.074 & & 5.974 \\
\hline $\mathrm{Mn}^{2+}$ & & 0.001 & 0.004 & 0.003 & 0.020 & 0.005 & 0.002 & 0.006 & 0.012 & 0.150 \\
\hline $\mathrm{Mg}^{2+}$ & 0.114 & & 0.814 & 0.186 & 1.284 & 0.049 & 0.003 & 0.696 & 1.530 & 1.184 \\
\hline $\mathrm{Ca}^{2+}$ & 0.013 & 0.017 & 0.945 & 0.296 & 0.022 & 0.338 & 0.253 & 0.943 & 0.015 & 0.245 \\
\hline $\mathrm{Na}^{1+}$ & 5.312 & 6.867 & 0.010 & 0.692 & & 6.113 & 2.352 & 0.035 & & \\
\hline $\mathrm{K}^{1+}$ & 2.177 & 0.493 & 0.004 & & & 1.104 & 1.372 & 0.001 & & \\
\hline $\mathrm{Cl}$ & & 2.021 & & & & & & & & \\
\hline Sum & 23.680 & 21.565 & 3.989 & 4.001 & 2.989 & 23.619 & 20.070 & 4.002 & 3.017 & 24.000 \\
\hline \multicolumn{11}{|l|}{ Cation ratio (\%) } \\
\hline $\mathrm{Mg}$ & & & 42.0 & 11.7 & 64.9 & & & 37.2 & 76.1 & 11.3 \\
\hline $\mathrm{Fe}$ & & & 9.3 & 44.8 & 35.0 & & & 12.3 & 23.9 & 73.1 \\
\hline $\mathrm{Ti}$ & & & & & & & & & & 15.6 \\
\hline $\mathrm{Ca}$ & 0.1 & 0.2 & 48.7 & 43.5 & 0.1 & 4.5 & 6.3 & 50.5 & 0.0 & \\
\hline $\mathrm{Na}$ & 70.8 & 93.2 & & & & 80.9 & 59.2 & & & \\
\hline $\mathrm{K}$ & 29.0 & 6.6 & & & & 14.6 & 34.5 & & & \\
\hline
\end{tabular}

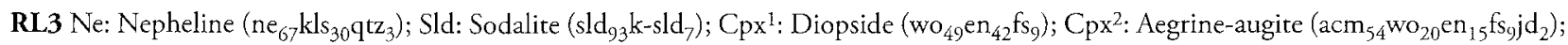
Ol: Forsrerite $\left(\mathrm{fo}_{65} \mathrm{fa}_{35}\right)$;

RL2A Ne: Nepheline $\left(\mathrm{ne}_{83} \mathrm{kl}_{15} \mathrm{qtz}_{22}\right)$; Afd: Anorthoclase $\left(\mathrm{ab}_{59} \mathrm{or}_{35} \mathrm{an}_{6}\right)$; Cpx: Diopside $\left(\mathrm{wo}_{51} \mathrm{en}_{37} \mathrm{fs}_{12}\right)$; Ol: Forsterite (fo $\left.\mathrm{f}_{76} \mathrm{fa}_{24}\right)$; Spl: Ulvospinel (usp60 $\mathrm{mag}_{19} \mathrm{mf}_{18} \mathrm{hc}_{1} \mathrm{chr}_{1}$ )

$\mathrm{Al}_{2} \mathrm{O}_{3} 1-7$ wt.\%). Ulvospinel (usp $42-61 \mathrm{mf}_{17-33} \operatorname{mag}_{8-20} \mathrm{hc}_{1-}$ ${ }_{14} \mathrm{Chr}_{1-4}$ ) forms sparse, large anhedral grains and is also present as inclusions in olivine $\left(\mathrm{fo}_{76-78}\right)$. Apatite is common as inclusions in the diopside.

\section{Syenitic pegmatoids}

These occur as nepheline-bearing and nepheline-free syenitic assemblages. The nepheline-bearing assemblages (RL2A, 7A, 11 ; table 4, pl. 2D) are dominated by coarse-grained, zoned subhedral to euhedral prismatic, pale brown to green rimmed, Ti-Al-bearing diopside $\left(\mathrm{wo}_{48-51} \mathrm{en}_{38-42} \mathrm{fs}_{10-12} ; \mathrm{TiO}_{2} 1.9-3.0\right.$ wt. $\%, \mathrm{Al}_{2} \mathrm{O}_{3} 3.0-6.5$ wt. $\left.\%\right)$. The central parts of these are crowded with inclusions, some being forsteritic olivine, and some outer rims pass into green sodic pyroxenes. The pyroxenes are embedded in coarse-grained, zoned sodic sanidine
( or $_{52-53} \mathrm{ab}_{4-47} \mathrm{an}_{1}$ ), subhedral nepheline ( $\mathrm{ne}_{81-83} \mathrm{kls}_{14-5} \mathrm{qtz}_{3-5}$ ) and ulvospinel (usp $47-55 \mathrm{mag}_{27-34} \mathrm{mf}_{19-34} \mathrm{hc}_{0-1} \mathrm{chr}_{0-1}$ ) grains. Subordinate mesostasis and brown glass contain apatite and traces of biotite.

Syenites lacking nepheline (RL15; table 4, pl. 2F) are relatively rare among the pegmatoids. Some contain orientated, partly radial, intergrowths of sanidine laths $\left(\right.$ or $_{48-52} \mathrm{ab}_{47-51} \mathrm{an}_{0-1}$ ) and elongate diopside blades $\left(w_{48-49} \mathrm{en}_{41-42} \mathrm{fs}_{9-10} ; \mathrm{TiO}_{2}\right.$ up to $\left.1.2 \mathrm{wt} . \%\right)$. The diopside develops outer rims and tips of more sodic green to dark green pyroxenes with compositions of aegirine-augite $\left.\left(\mathrm{acm}_{23-38}\right) \mathrm{j}_{0-2} \mathrm{Cf}_{0-2} \mathrm{wO}_{29-35} \mathrm{en}_{22-29} \mathrm{fs}_{9-11}\right)$ and aegirine $\left(\mathrm{acm}_{78-83} \mathrm{jd}_{2-3} \mathrm{wo}_{11} \mathrm{en}_{6-10} \mathrm{fs}_{0}\right)$. Accessory green-brown amphibole grains range from titanian richterite and titanian ferro-richterite to potassic-titanian-richterite and titanianmagnesiokatophorite. 
TABLE 4

Representative mineral analyses, ne-syenitoid and syenitoids

\begin{tabular}{|c|c|c|c|c|c|c|c|c|c|c|}
\hline \multirow{2}{*}{$\begin{array}{l}\text { Analysis wt.\% } \\
\text { Mineral }\end{array}$} & \multicolumn{5}{|c|}{ Nepheline syenite (RL6) } & \multicolumn{5}{|c|}{ Alkali syenite (RL15) } \\
\hline & Afd & $\mathrm{Ne}$ & Cpx & Spl & $\mathrm{Ol}$ & Afd & $\mathrm{Cpx}$ & $\mathrm{Cpx}^{2}$ & $\mathrm{Cpx}^{3}$ & Amp \\
\hline $\mathrm{SiO}_{2}$ & 66.29 & 44.06 & 51.31 & 0.66 & 38.62 & 65.80 & 53.98 & 51.34 & 50.65 & 50.84 \\
\hline $\mathrm{TiO}_{2}$ & 0.24 & & 2.15 & 15.95 & & 0.32 & 0.26 & 1.38 & 3.31 & 4.76 \\
\hline $\mathrm{Al}_{2} \mathrm{O}_{3}$ & 18.75 & 33.51 & 3.04 & & 0.22 & 18.12 & 0.44 & 0.33 & 0.36 & 1.21 \\
\hline $\mathrm{Cr}_{2} \mathrm{O}_{3}$ & & & 0.19 & & & & & & & \\
\hline $\mathrm{FeO}$ & 0.72 & 0.88 & 6.13 & 40.30 & 22.01 & 1.56 & 3.92 & 5.49 & 0.00 & 14.33 \\
\hline $\mathrm{Fe}_{2} \mathrm{O}_{3}$ & & & & 38.44 & & & 2.37 & 12.88 & 26.35 & \\
\hline $\mathrm{MnO}$ & & & & 1.03 & 0.46 & & 0.20 & 0.31 & 0.15 & 0.16 \\
\hline $\mathrm{MgO}$ & & & 13.94 & 3.38 & 38.46 & 0.12 & 15.07 & 7.82 & 3.35 & 13.15 \\
\hline $\mathrm{CaO}$ & 0.18 & 0.54 & 23.17 & 0.15 & 0.82 & 0.04 & 24.38 & 14.25 & 5.04 & 4.97 \\
\hline $\mathrm{Na}_{2} \mathrm{O}$ & 5.31 & 16.93 & 0.56 & & & 5.47 & 0.54 & 5.26 & 11.39 & 6.92 \\
\hline $\mathrm{K}_{2} \mathrm{O}$ & 8.99 & 4.36 & & & & 8.55 & 0.02 & 0.08 & 0.03 & 1.38 \\
\hline Total & 100.48 & 100.29 & 100.49 & 99.91 & 100.59 & 99.98 & 100.94 & 99.14 & 100.63 & 97.72 \\
\hline Cations (No. O) & 32 & 32 & 6 & 32 & 4 & 32 & 6 & 6 & 6 & 23 \\
\hline $\mathrm{Si}^{4+}$ & 11.932 & 8.396 & 1.892 & 0.195 & 0.999 & 11.937 & 1.981 & 1.964 & 1.911 & 7.514 \\
\hline $\mathrm{Ti}^{4+}$ & 0.032 & 7.528 & 0.060 & 3.538 & & 0.044 & 0.007 & 0.040 & 0.094 & 0.529 \\
\hline $\mathrm{Al}^{3+}$ & 3.980 & 0.140 & 0.132 & & 0.007 & 3.874 & 0.019 & 0.015 & 0.016 & 0.211 \\
\hline $\mathrm{Cr}^{3+}$ & & & 0.006 & & & & & & & \\
\hline $\mathrm{Fe}^{2+}$ & 0.018 & & 0.189 & 9.942 & 0.476 & 0.237 & 0.120 & 0.176 & 0.000 & 1.771 \\
\hline $\mathrm{Fe}^{3+}$ & & & & 8.534 & & & 0.065 & 0.371 & 0.748 & 0.000 \\
\hline $\mathrm{Mn}^{2+}$ & & & & 0.257 & 0.010 & & 0.006 & 0.010 & 0.005 & 0.020 \\
\hline $\mathrm{Mg}^{2+}$ & & & 0.766 & 1.486 & 1.483 & 0.032 & 0.820 & 0.446 & 0.188 & 20897 \\
\hline $\mathrm{Ca}^{2+}$ & 0.036 & 0.112 & 0.915 & 0.047 & 0.023 & 0.008 & 0.954 & 0.584 & 0.204 & 0.787 \\
\hline $\mathrm{Na}^{1+}$ & 0.463 & 6.256 & 0.040 & & & 1.924 & 0.038 & 0.390 & 0.833 & 1.983 \\
\hline $\mathrm{K}^{1+}$ & 1.852 & 1.060 & & & & 1.979 & 0.001 & 0.004 & 0.001 & 0.260 \\
\hline Sum & 20.004 & 23.496 & 4.000 & 27.455 & 2.997 & 20.035 & 4.001 & 4.000 & 4.000 & 15.972 \\
\hline Cation ratio (\%) & & & & 23.981 & & & & & & \\
\hline $\mathrm{Mg}$ & & & 41.0 & 6.3 & 74.9 & & 41.6 & 28.4 & 11.4 & 43.7 \\
\hline $\mathrm{Fe}$ & & & 10.1 & 78.5 & 24.0 & & 9.6 & 34.6 & 41.9 & 26.4 \\
\hline $\mathrm{Ti}$ & & & & 15.1 & & & & & & \\
\hline $\mathrm{Ca}$ & 0.9 & 1.5 & 48.9 & & 1.1 & 0.3 & 48.7 & 37.0 & & \\
\hline $\mathrm{Na}$ & 46.9 & 84.2 & & & & 49.2 & & & 46.7 & 29.9 \\
\hline $\mathrm{K}$ & 52.2 & 14.3 & & & & 50.5 & & & & \\
\hline
\end{tabular}

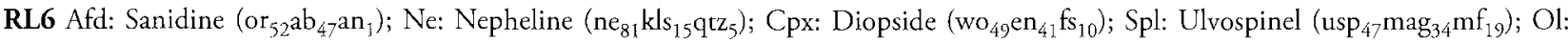
Forsterite $\left(\mathrm{fo}_{75} \mathrm{fa}_{5}\right)$.

RL15 Afd: Sanidine $\left(\mathrm{or}_{51} \mathrm{ab}_{49} \mathrm{an}_{0}\right) ; \mathrm{Cpx}{ }^{1}$ : Diopside $\left(w_{49} \mathrm{en}_{42} \mathrm{fs}_{10}\right) ; \mathrm{Cpx}^{2}$ : Aegirine-augite $\left(\operatorname{acm}_{37} \mathrm{wo}_{29} \mathrm{en}_{22} \mathrm{fs}_{9} \mathrm{jd}_{2}\right) ; \mathrm{Cpx}^{3}:$ Aegirine $\left(\right.$ acm $\left._{78} \mathrm{wo}_{11} \mathrm{en}_{10} \mathrm{jd}_{2} \mathrm{fs}_{0}\right) ;$ Amp: Potassian-titanian-richterite.

\section{Mantle meta-assemblages}

These coarse-grained metamorphic assemblages are mostly spinel meta-lherzolite, but include spinel meta-wehrlite and spinel meta-websterite. The spinel meta-lherzolites (RL9, RL18, table 5, pl. 2G) consist of anhedral aggregates of olivine (fo f8-89 $)$, Al-Cr-bearing diopside $\left(w_{50-52} \mathrm{en}_{44-46} \mathrm{fs}_{4-5}\right.$; $\mathrm{Al}_{2} \mathrm{O}_{3} 5.0-5.2$ wt. $\%, \mathrm{Cr}_{2} \mathrm{O}_{3} 0.8-1.0$ wt. $\%$ ) and Al-bearing enstatite $\left(\mathrm{en}_{88-89} \mathrm{fs}_{10-11} \mathrm{wo}_{1}\right.$ ), interspersed with accessory $\mathrm{Cr}$ bearing spinel $\left(\mathrm{spl}_{70} \mathrm{Chr}_{14} \mathrm{hc}_{7}\right)$. The enstatite includes blebs and lamellae of Al-bearing diopside $\left(\mathrm{wo}_{48-50} \mathrm{en}_{45-47} \mathrm{fs}_{5} ; \mathrm{Al}_{2} \mathrm{O}_{3} 5\right.$ wt. \%), which gave estimated metamorphic re-equilibration temperatures of $890-950^{\circ} \mathrm{C}$ (Wells 1977 , two-pyroxene $\mathrm{Fe}^{2+}$ thermometry).

Spinel meta-wehrlites (RL6A; table 5; pl. 2H) contain anhedral olivine $\left(\mathrm{fo}_{88-89}\right)$ and $\mathrm{Cr}$-Al-bearing diopside $\left(\mathrm{wO}_{49-}\right.$ ${ }_{50} \mathrm{en}_{46-47} \mathrm{fs}_{4} ; \mathrm{Al}_{2} \mathrm{O}_{3} 1.3-2.3$ wt. $\%, \mathrm{Cr}_{2} \mathrm{O}_{3} 1.0-1.1$ wt. \%) and some interstitial Cr-bearing spinel ( $\left.\mathrm{spl}_{73} \mathrm{Chr}_{10} \mathrm{hc}_{9} \mathrm{mag}_{6}\right)$. The diopsides are spongy and partly replaced by melting reactants.

Many of the metamorphic assemblages show considerable recrystallisation and alteration related to reactions with fluid infiltrations from the host nephelinite. Recrystallisation is accompanied by replacement of the coarser phases by small olivine, clinopyroxene and ulvospinel grains, formation of sporadic large anhedral green spinel grains and development of a glassy melt. Initial reaction takes place along grain boundaries, but become more complex as clinopyroxenes and spinels develop sieve texture and orthopyroxene reacts to form replacement rims and glassy melt textures. Similar reactants were described in mantle suites in Boat Harbour, NW Tasmania, olivine nephelinite (Sutherland et al. 1996) and the compositions of such products have been reproduced experimentally at low pressure (Shaw \& Dingwell 2008). 
TABLE 5

Representative mineral analyses, meta-peridotites

\begin{tabular}{|c|c|c|c|c|c|c|c|c|c|c|}
\hline \multirow{2}{*}{$\begin{array}{l}\text { Analysis wt.\% } \\
\text { Mineral }\end{array}$} & \multicolumn{7}{|c|}{ Spinel lherzolite (RL9) } & \multicolumn{3}{|c|}{ Meta-wehrlite (RL6A) } \\
\hline & $\mathrm{Ol}$ & Cpx & $\mathrm{Opx}^{1}$ & $\mathrm{Cpx^{1 }}$ & $\mathrm{Opx}^{2}$ & $\mathrm{Cpx}^{2}$ & Spl & $\mathrm{Ol}$ & $\mathrm{Cpx}$ & $S p l$ \\
\hline $\mathrm{SiO}_{2}$ & 41.19 & 52.20 & 55.23 & 52.06 & 54.93 & 52.06 & & 40.70 & 54.31 & 0.37 \\
\hline $\mathrm{TiO}_{2}$ & 0.10 & 0.54 & 0.13 & 0.60 & 0.14 & 0.63 & 0.18 & & 0.21 & 0.16 \\
\hline $\mathrm{Al}_{2} \mathrm{O}_{3}$ & & 5.03 & 3.67 & 4.98 & 3.76 & 5.14 & 56.29 & & 1.28 & 53.49 \\
\hline $\mathrm{Cr}_{2} \mathrm{O}_{3}$ & & 1.03 & 0.34 & 0.72 & 0.37 & 0.83 & 13.38 & & 1.00 & 9.97 \\
\hline $\mathrm{FeO}$ & 11.05 & 2.78 & 6.70 & 2.97 & 6.72 & 2.96 & 9.95 & 10.72 & 2.66 & 12.49 \\
\hline $\mathrm{Fe}_{2} \mathrm{O}_{3}$ & & & & & & & 0.23 & & & 5.40 \\
\hline $\mathrm{MnO}$ & 0.19 & & 0.14 & 0.08 & 0.25 & 0.02 & 0.03 & 0.22 & 0.07 & 0.29 \\
\hline $\mathrm{MgO}$ & 47.31 & 14.67 & 31.77 & 15.46 & 31.51 & 15.16 & 20.44 & 48.38 & 16.34 & 18.74 \\
\hline $\mathrm{CaO}$ & & 22.59 & 0.43 & 22.34 & 0.39 & 22.53 & & & 24.55 & \\
\hline $\mathrm{Na}_{2} \mathrm{O}$ & & 1.02 & & 0.48 & & 0.76 & & & 0.15 & 0.01 \\
\hline $\mathrm{K}_{2} \mathrm{O}$ & & & & & & 0.04 & & & 0.04 & \\
\hline Total & 99.84 & 99.86 & 98.41 & 99.69 & 98.07 & 100.13 & 100.50 & 100.02 & 100.62 & 100.92 \\
\hline Cations (No. O) & 4 & 6 & 6 & 6 & 6 & 6 & 32 & 4 & 6 & 32 \\
\hline $\mathrm{Si}^{4+}$ & 1.014 & 1.902 & 1.937 & 1.897 & 1.943 & 1.892 & & 1.001 & 1.974 & 0.078 \\
\hline $\mathrm{Ti}^{4+}$ & 0.002 & 0.015 & 0.003 & 0.016 & 0.004 & 0.017 & 0.028 & & 0.006 & 0.025 \\
\hline $\mathrm{Al}^{3+}$ & & 0.216 & 0.152 & 0.214 & 0.156 & 0.220 & 13.721 & & 0.055 & 13.215 \\
\hline $\mathrm{Cr}^{3+}$ & & 0.030 & 0.009 & 0.021 & 0.010 & 0.024 & 2.188 & & 0.029 & 1.652 \\
\hline $\mathrm{Fe}^{2+}$ & 0.228 & 0.085 & 0.197 & 0.091 & 0.198 & 0.090 & 1.721 & 0.220 & 0.081 & 2.190 \\
\hline $\mathrm{Fe}^{3+}$ & & & & & & & 0.035 & & & 0.930 \\
\hline $\mathrm{Mn}^{2+}$ & 0.004 & & 0.004 & & 0.008 & 0.001 & 0.005 & 0.005 & 0.002 & 0.052 \\
\hline $\mathrm{Mg}^{2+}$ & 1.737 & 0.797 & 1.661 & 0.840 & 1.663 & 0.821 & 6.302 & 1.773 & 0.883 & 5.856 \\
\hline $\mathrm{Ca}^{2+}$ & & 0.882 & 0.016 & 0.872 & 0.015 & 0.877 & & & 0.954 & \\
\hline $\mathrm{Na}^{1+}$ & & 0.072 & & 0.034 & & 0.054 & & & 0.011 & \\
\hline $\mathrm{K}^{1+}$ & & & & & & 0.002 & & & 0.002 & 0.003 \\
\hline Sum & 2.985 & 3.999 & 3.979 & 3.99 & 3.997 & 3.998 & 24.000 & 2.999 & 3.997 & 24.000 \\
\hline Cation ratio (\% & & & & & & & & & & \\
\hline $\mathrm{Mg}$ & 88.3 & 45.5 & 88.5 & 46.7 & 88.7 & 45.8 & 61.5 & 88.9 & 46.1 & 55.1 \\
\hline $\mathrm{Fe}$ & 11.7 & 4.5 & 10.6 & 5.0 & 10.5 & 5.0 & 17.1 & 11.1 & 4.2 & 29.3 \\
\hline $\mathrm{Ca}$ & 0.0 & 50.0 & 1.1 & 48.3 & 0.8 & 49.2 & & 0.0 & 49.7 & \\
\hline $\mathrm{Cr}$ & & & & & & & 21.3 & & & 15.6 \\
\hline
\end{tabular}

RL9. Ol: Forsterite $\left(\mathrm{fo}_{88} \mathrm{fa}_{12}\right)$; Cpx: Diopside $\left(\mathrm{wo}_{50} \mathrm{en}_{46} \mathrm{fs}_{5}\right) ; \mathrm{Opx}^{-1}$ : Enstatite $\left(\mathrm{en}_{89} \mathrm{fs}_{11} \mathrm{wo}_{1}\right) ; \mathrm{Cpx}^{1}$ : Diosipde $\left(\mathrm{wo}_{48} \mathrm{en}_{47} \mathrm{fs}_{5}\right) ; \mathrm{Opx}^{2}$ : Estatite

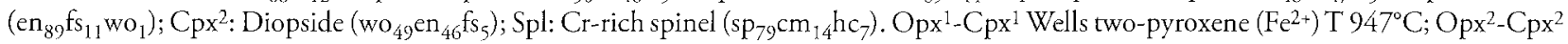
Wells two-pyroxene $\left(\mathrm{Fe}^{2+}\right) \mathrm{T} 890^{\circ} \mathrm{C}$

RL6A Ol: Forsterite ( $\left.\mathrm{fo}_{89} \mathrm{fa}_{11}\right)$; Cpx: Diopside ( $\left.\mathrm{wo}_{50} \mathrm{en}_{46} \mathrm{fs}_{4}\right)$; Spl: spinel ( $\left.\mathrm{spl}_{73} \mathrm{chr}_{10} \mathrm{hc}_{9} \mathrm{mag}_{6} \mathrm{usp} \mathrm{p}_{6}\right)$.

\section{DISCUSSION}

The Round Lagoon nephelinite and contained coarse assemblages suggest its magmatic evolution took place under at least two different pressure-temperature regimes. The cumulate wehrlite and ol-clinopyroxenite bodies in the mantle meta-lherzolite xenolich (fig. 1) suggests a higher pressure, shallow mantle stage. Other cognate ultramafic to mafic assemblages, however, have mineral compositions similar to those in the host nephelinite that relate to later, lower pressure crystallisation.

\section{Host nephelinite}

This is a K-rich olivine nephelinite with a low ${ }^{87} \mathrm{Sr} /{ }^{86} \mathrm{Sr}$ $(0.7302 \pm 3)$ and is isotopically typical of many Tasmanian undersaturated alkaline lavas (McDonough et al. 1985, Sutherland 1989). In the spectrum of strongly undersaturated alkaline lavas in Tasmania, it has high $\mathrm{SiO}_{2}$ content suggesting transition into basanite (fig. 2). The analysis has a high, near-

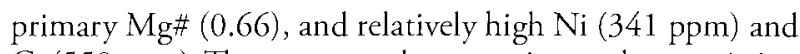
$\mathrm{Cr}(550 \mathrm{ppm})$. These apparently near-primary characteristics, however, are probably misleading due to many inextricable small xenocrysts of olivine and pyroxene, thus the rock does not represent a liquid composition. The magma was probably mildly fractionated, based on its olivine phenocryst compositions (up to $\mathrm{fo}_{78}$ which would be in equilibrium with a liquid of $\mathrm{Mg} \# 0.51-0.52$ ) and its relatively elevated incompatible elements; for example it has a $\mathrm{Zr}$ content (460 ppm) compared to $\mathrm{Zr}$ contents ( $<370 \mathrm{ppm})$ in other primary olivine nephelinites in Tasmania (Sutherland 1989, Sutherland et al. 1996, Sutherland et al. 2004, J. Everard, unpubl. data base). Available trace element data normalised to primitive mantle contents (fig. 3) compare closely with patterns for a fractionated olivine nephelinite $(\mathrm{Mg \# 0.59)}$ and mantle-derived basanite (UT704489) from the Midlandsarea, and are higher in most of these elements than nephelinites from Boat Harbour. Based on the occurrence of the cumulate wehrlite and olivine clinopyroxenite found within the mantle xenolith suite, such fractionation was probably initiated at 


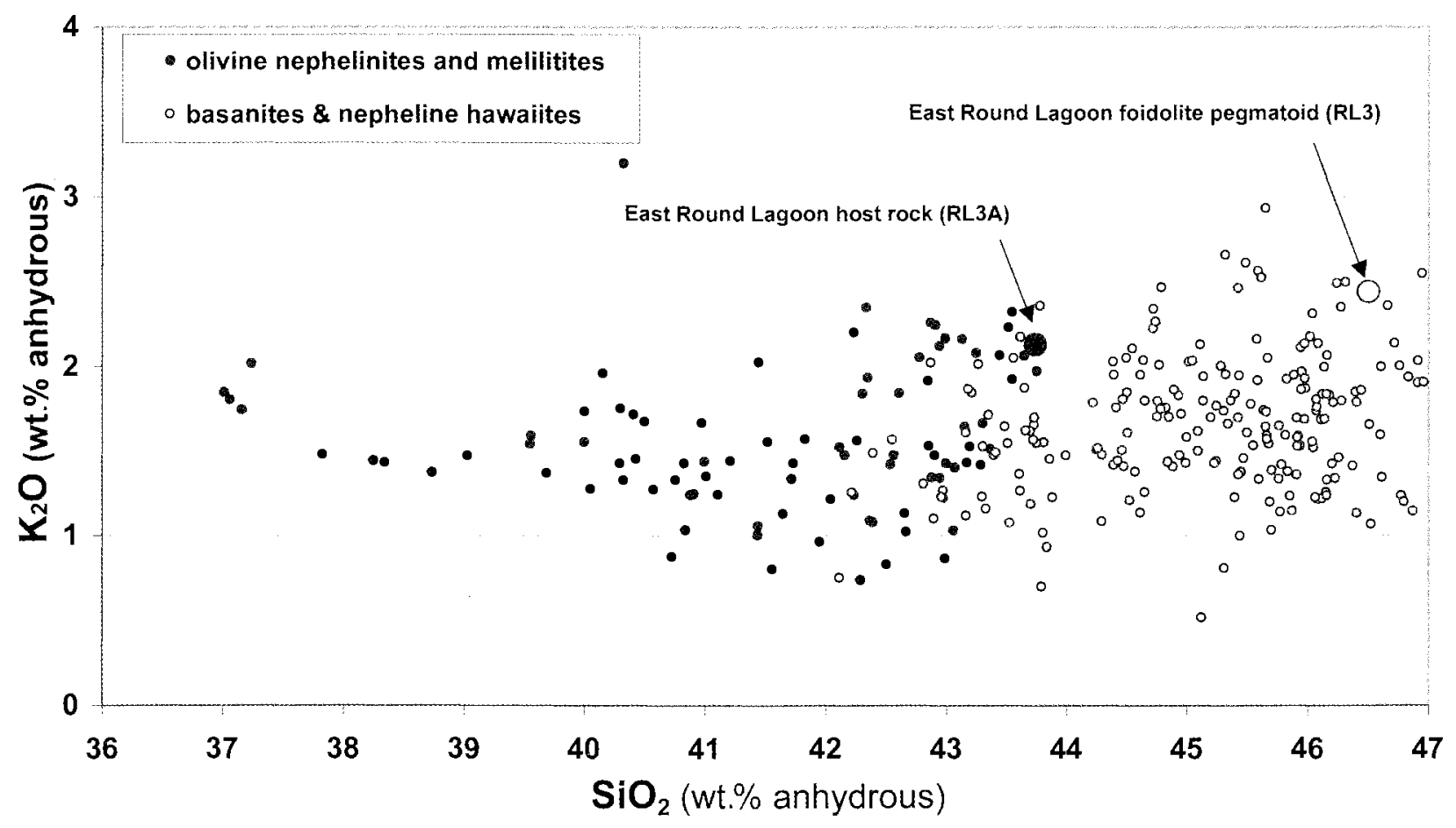

FIG. 2 - Alkali $\left(\mathrm{K}_{2} \mathrm{O}\right)-\mathrm{SiO}_{2}$ (anhydrous) plots for Tasmanian melilitites and nephelinites, in relationship to the Round Lagoon nephelinite and sodalite ijolite (larger circles).

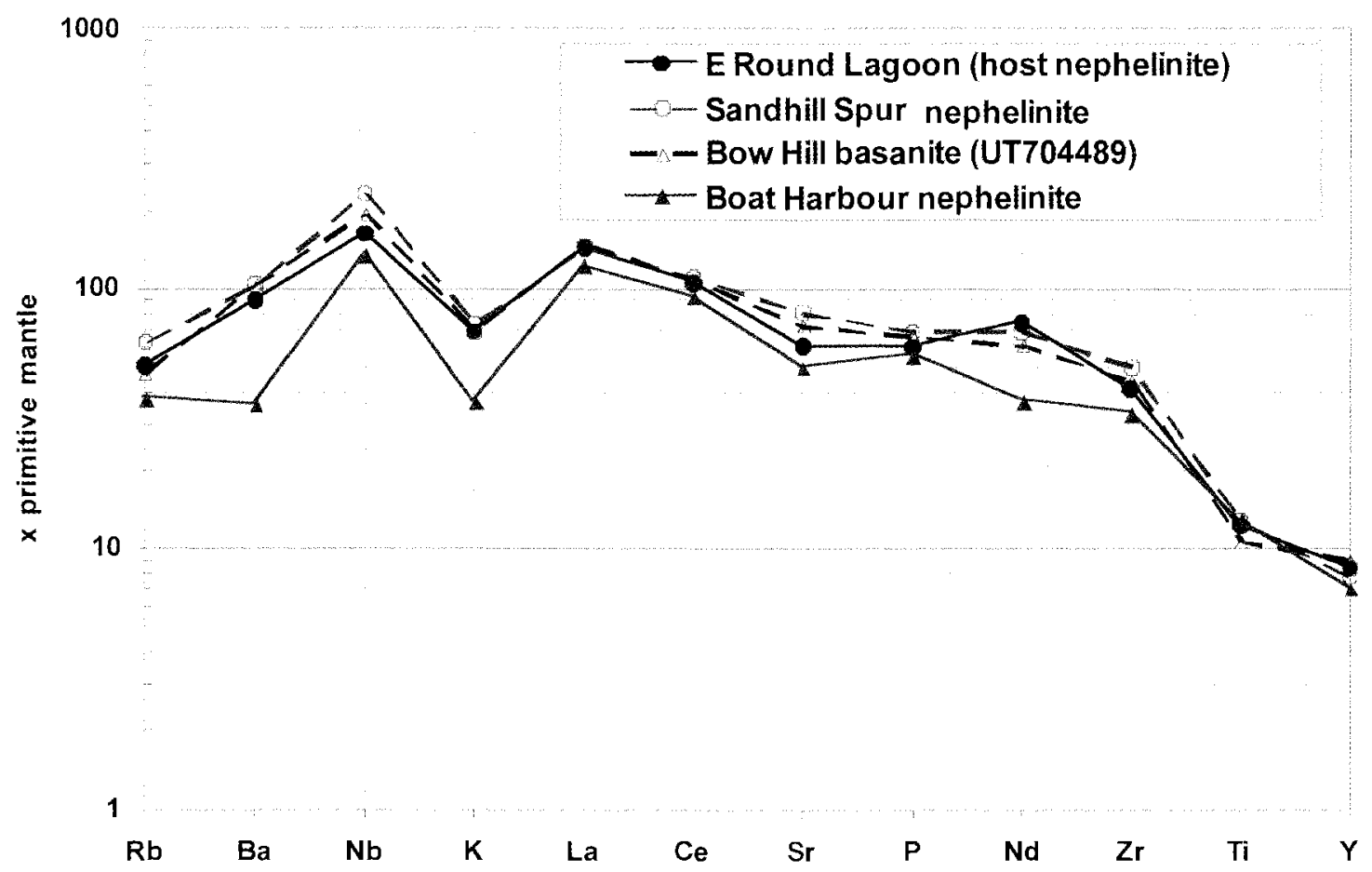

FIG. 3 - Incompatible multi-element plots for Round Lagoon nephelinite in comparison to other Tasmanian nephelinites and transitional basanites. Normalising factors are after Sun \& McDonough (1989). Other data sources: Sandhill Spur (Sutherland et al. 2004), Bow Hill (Adam 1990) and Boat Harbour (Sutherland et al. 1996). 
mantle depths under the site, although detailed rare earth element and isotopic analyses of both host and cumulate xenoliths are needed to confirm this. The mantle peridotites indicate a fast ascent of the nephelinite host, probably in the range of $10^{-2}$ to $5 \mathrm{~m} / \mathrm{s}$ (Spera 1984). A primary composition of the original nephelinite magma could be provisionally estimated by adding in $\mathrm{Mg}$-rich olivine, clinopyroxene \pm spinel compositions found in wehrlite cumulates within the host nephelinite (table 2).

As proportions of such liquidus phases are only broadly constrained, two potential extracts were calculated; one had abundant olivine (65\%), moderate clinopyroxene (30\%) and accessory spinel $(5 \%)$; the other had clinopyroxene $(65 \%)$ dominant over olivine (30\%), with accessory spinel $(5 \%)$. The mineral compositions added from table 2 give potential magma compositions at $10 \%$ and $20 \%$ liquidus crystallisation. The olivine-dominant scheme gave $\mathrm{Mg}$ \# 0.73 and 0.76 respectively, with high normative ol (24$28 \%)$, di (27-28\%) and ne (13-15\%) and or (10\%). The clinopyroxene-dominant scheme gave $\mathrm{Mg} \# 0.72$ and 0.74 respectively, lesser normative ol $(21-28 \%)$ to di $(30-35 \%)$, but similar ne (14-15\%) and or (10-12\%) ranges. The latter parents resemble primitive mantle-derived olivine nephelinite (Mg \# 0.73-0.75) in young Tasman Sea volcanism (Briggs et al. 1997).

The above calculated parent compositions would be partly modified under mantle conditions, in which more Al-Narich clinopyroxene typifies high-pressure liquidus phases. In ne-rich rocks and experimental runs on Tasmanian and other nephelinites such clinopyroxenes contain up to 10 wt. $\% \mathrm{Al}_{2} \mathrm{O}_{3}, 4$ wt. $\% \mathrm{TiO}_{2}$ and 2 wt. $\% \mathrm{Na}_{2} \mathrm{O}$ (Bultitude \& Green 1971, Fodor et al. 1982, Adam 1990, Sutherland et al. 1996, 2004, 2005, Woodland \& Jugo 2007). Thus, a Round Lagoon mantle parent would have higher $\mathrm{Al}, \mathrm{Ti}$ and $\mathrm{Na}$ than estimated from the low-pressure wherlitic extracts, but would be compatible with a high-pressure fractionation series in which sodic nephelinite magmas evolve to more K-rich members (Sutherland 1974). The Round Hill nephelinite, when adjusted for hydrous content, is near a Bow Hill, Tasmanian basanite in composition, which experimentally crystallised clinopyroxene at $\mathrm{P}>2.6$ $\mathrm{GPa}$ and clinopyroxene and olivine at lower pressures in runs at $1270^{\circ} \mathrm{C}$ and 4.5 wt. $\% \mathrm{H}_{2} \mathrm{O}$ (UT-70489, Adam 1990). Based on such experimental work (Adam 1990, Green \& Falloon 1998), the Round Lagoon magma probably segregated at $3 \mathrm{GPa}(90 \mathrm{~km}$ depth) under low degrees of partial melting of garnet lherzolite that contained $<7 \mathrm{wt} . \%$ volatiles $\left(\mathrm{H}_{2} \mathrm{O}+\mathrm{CO}_{2}\right)$. The nephelinite has high $\mathrm{Ba}$, and to a lesser degree high $\mathrm{Zr}, \mathrm{Nb}$, and $\mathrm{K}$. The $\mathrm{Ba} / \mathrm{Zr}_{\mathrm{r}}(1.4)$ and $\mathrm{Ba} /$ $\mathrm{Nb}$ (5.4) ratios are relatively high compared to some other Tasmanian nephelinites $(\mathrm{Ba} / \mathrm{Zr}<1, \mathrm{Ba} / \mathrm{Nb}>3)$; which suggests either amphibole was low in the mantle source (Chazot et al. 1996), or that its contribution to the magma became diluted through the suspected mantle fractionation.

The lack of garnet or its replacements in Round Lagoon inclusions suggests the magma stalled and fractionated at mantle levels shallower than the spinel-garnet lherzolite transition, i.e., <1.7-1.8 GPa and $1100-1200^{\circ} \mathrm{C}$ for Tasmanian Miocene geotherms (Sutherland et al. 2005). The two-pyroxene temperatures for Round Lagoon spinel metaIherzolite reach $950^{\circ} \mathrm{C}$ (table 5). Based on the Tasmanian geotherm, this suggests magma began to fractionate at around 1.0-1.1 GPa $(30-35 \mathrm{~km})$, at mantle depths shallower than the spinel-garnet pyroxenite transition. This agrees with a Moho depth at $30 \mathrm{~km}$ below the area. At these pressures, using the calculated $\mathrm{CaO} /(\mathrm{CaO}+\mathrm{MgO})$ ratio of the nephelinite parental magma, the liquidus phases crystallising during this mantle fractionation would favour significant olivine along with clinopyroxene (Smith et al. 2008).

\section{Low-pressure crystallisation}

Clinopyroxenes in the Round Lagoon cumulate and pegmatoid assemblages mostly show relatively high $\mathrm{Al}^{\mathrm{IV}} / \mathrm{Al}^{\mathrm{VI}}$ ratios (1.5-13.5). Such ratios'typically indicate low-pressure igneous crystallisation, rather than mantle and lower crust pressures (Wass 1979, Mason 1985, Simonetti et al. 1996). The mantle xenolith clinopyroxenes give ratios of 1 or less, but some from wehrlitic cumulates have transitional values. Amphiboles in the pegmatoids also show high AlIV and Ti, features that were found in lower pressure experimental runs of strongly undersaturated magmas (Adam et al. 2007).

\section{Uitramafic crystallisation}

Initial temperatures for crystallising liquidus minerals from such nephelinite magmas are about $1300^{\circ} \mathrm{C}$ for olivine, $1150^{\circ} \mathrm{C}$ for clinopyroxene and $1065^{\circ} \mathrm{C}$ for nepheline (Wilkinson 1977). These intervals cover the wehrlite (RL8) and ol-ne-bearing clinopyroxenite (RL19) assemblages. Olivine is highly magnesian $\left(\mathrm{fo}_{90-91}\right)$ in the wehrlites but less so in the clinopyroxenites $\left(\mathrm{fo}_{73}\right)$; the latter contains minor $\mathrm{Ca}_{2} \mathrm{SiO}_{4}$ and $\mathrm{Mn}_{2} \mathrm{SiO}_{4}$ end member components. Clinopyroxenes are $\mathrm{Na}-\mathrm{Cr}$-enriched augites in the wehrlites, but these elements are lower, and $\mathrm{Ca}$ and $\mathrm{Fe}$ higher in the diopside of the clinopyroxenites. Early nepheline in the clinopyroxenites is sodic ( ne $_{76-77}$ ), and compositions suggest crystallisation around $980^{\circ} \mathrm{C}$, based on experimental isotherms (Hamilton 1961). Estimates for crystallisation temperatures from nephelines, however, may be minimum values due to potential exchanges of alkalis and aqueous vapour that reset them to lower kls and qtz and so apparently lower temperatures (Edgar 1984).

Residual glass in the clinopyroxenites has an alkaline trachytic composition (table 6). Normatively this corresponds to minor Q (3\%), Ab-rich plagioclase (43\%) and $\mathrm{Or}(47 \%)$, together with accessory Hy, C, Mt and Il. Removal of $\mathrm{MgO}(1.9$ wt.\%) and $\mathrm{FeO}(1.2$ wt.\%) in the glass to crystallise spinel, leaves a remainder close to alkali feldspar in composition, with a stoichiometric formula approximating anorthoclase $\left(\mathrm{ab}_{77} \mathrm{Or}_{21} \mathrm{an}_{2}\right)$. A potential spinel-bearing, anorthoclase-rich crystallisation from this glass is compatible with such phases observed in the more evolved pegmatoid (RL2A).

\section{Main mafic crystallisation}

Depletion of $\mathrm{Mg}, \mathrm{Ca}$ and $\mathrm{Fe}$ and accumulation of alkalis and volatiles in the magma then produced sodic feldspathoidalrich assemblages, such as sodalite-ijolite (RL3). Nepheline $\left(\mathrm{Ne}_{67-68}\right)$ in the sodalite-ijolites (RL3) is richer in $\mathrm{K}$ than in the nepheline-dominant ijolite (RL2A) and syenite (RL6) (fig. 4), as crystallising sodalite would compete for $\mathrm{Na}$. Its composition suggests formation near $700^{\circ} \mathrm{C}$ based on experimental isotherms (Hamilton 1961). With up to $20 \%$ sodalite crystallisation, $\mathrm{Cl}$ was a significant volatile in the system. Crystallising pyroxene was accordingly $\mathrm{Na}$ poor diopside, but incorporated $\mathrm{Fe}$, $\mathrm{Ti}$ and $\mathrm{Na}$ to form late aegirine-augite $\left(\mathrm{acm}_{53-57}\right)$.

As feldspathoid crystallisation diminished, alkali feldspar $\left(a b_{59-61}\right.$ or $\left._{33-35} a_{6-7}\right)$ became more important (fig. 4) in ijolitic assemblages (RL2A). Nepheline became more 
TABLE 6

Major and trace element analyses, nephelinite host, foidolite and cumulate glass

\begin{tabular}{|c|c|c|c|c|c|c|}
\hline \multirow{2}{*}{$\begin{array}{l}\text { Analysis } \\
\text { Oxides (wt.\%) }\end{array}$} & \multicolumn{3}{|c|}{ Major elements, CIPW norms and Indices } & \multicolumn{3}{|c|}{ Trace elements (ppm) } \\
\hline & Nephelinite $^{1}$ & Foidolite $^{1}$ & Glass $^{2}$ & \multicolumn{2}{|c|}{ Nephelinite $^{1}$} & \multirow{2}{*}{ Foidolite ${ }^{1}$} \\
\hline $\mathrm{SiO}_{2}$ & 42.28 & 45.95 & 64.59 & $\mathrm{Cr}$ & 550 & \\
\hline $\mathrm{TiO}_{2}^{2}$ & 2.65 & 2.50 & 0.20 & $\mathrm{Ni}$ & 341 & 125 \\
\hline $\mathrm{Al}_{2} \mathrm{O}_{3}$ & 10.81 & 13.03 & 17.36 & $\mathrm{~V}$ & 112 & 290 \\
\hline $\mathrm{Fe}_{2} \mathrm{O}_{3}$ & 3.94 & 4.92 & 0.21 & & & \\
\hline $\mathrm{FeO}$ & 8.61 & 4.57 & 1.03 & $\mathrm{Ba}$ & 625 & 1100 \\
\hline $\mathrm{MnO}$ & 0.20 & 0.15 & & Y & 38 & 38 \\
\hline $\mathrm{MgO}$ & 11.35 & 7.95 & 1.90 & $\mathrm{Sr}$ & 1269 & 1850 \\
\hline $\mathrm{CaO}$ & 9.83 & 12.67 & 0.17 & $\mathrm{Zr}$ & 460 & 520 \\
\hline $\mathrm{Na}_{2} \mathrm{O}$ & 4.03 & 3.89 & 4.88 & $\mathrm{U}$ & $<2$ & $<5$ \\
\hline $\mathrm{K}_{2} \mathrm{O}$ & 2.06 & 2.41 & 7.81 & $\mathrm{Rb}$ & 32 & 68 \\
\hline $\mathrm{P}_{2} \mathrm{O}_{5}$ & 1.30 & 1.26 & & Th & 9 & $<4$ \\
\hline $\mathrm{H}_{2} \mathrm{O}_{+}$ & 2.49 & 0.14 & & $\mathrm{~Pb}$ & 27 & 8 \\
\hline $\mathrm{CO}_{2}$ & 0.28 & 0.37 & & $\mathrm{Ga}$ & 24 & 19 \\
\hline Total & 99.83 & 99.81 & 98.15 & $\mathrm{Nb}$ & 115 & 210 \\
\hline $\mathrm{Na}_{2} \mathrm{O} / \mathrm{K}_{2} \mathrm{O}$ & 1.96 & 4.10 & 0.62 & & & \\
\hline \multicolumn{4}{|c|}{ CIPW Norm (anhydrous, based on $\mathrm{Fe}_{2} \mathrm{O}_{3} / \mathrm{FeO}=0.2$ ) } & $\mathrm{La}$ & $\begin{array}{r}98 \\
187\end{array}$ & NA \\
\hline Q & & & 2.53 & $\mathrm{Ce}$ & $\begin{array}{l}187 \\
100\end{array}$ & $\begin{array}{l}\mathrm{NA} \\
\mathrm{NA}\end{array}$ \\
\hline $\mathrm{Or}$ & 12.56 & 14.38 & 47.02 & & & \\
\hline $\mathrm{Ab}$ & 3.36 & 5.18 & 42.07 & $\mathrm{Zn}$ & 175 & 93 \\
\hline An & 5.49 & 11.08 & 0.86 & $\mathrm{Cu}$ & 57 & 70 \\
\hline $\mathrm{Ne}$ & 17.24 & 15.20 & 6.24 & & & \\
\hline Di & 29.14 & 33.59 & & \multicolumn{3}{|c|}{ Trace element ratios } \\
\hline $\mathrm{Ol}$ & 20.77 & 8.53 & & $\mathrm{Cr} / \mathrm{Ni}$ & 1.613 & 0.689 \\
\hline $\mathrm{Mt}$ & 3.08 & 2.23 & 0.31 & $\mathrm{Rb} / \mathrm{Sr}$ & 0.025 & 0.037 \\
\hline Il & 5.17 & 4.80 & 0.39 & $\mathrm{Zr} / \mathrm{Nb}$ & 4.000 & 2.477 \\
\hline Ap & 3.16 & 3.16 & & $\mathrm{Ba} / \mathrm{Nb}$ & 5.435 & 5.238 \\
\hline Other & & & 0.58 & $\mathrm{Rb} / \mathrm{Nb}$ & 0.278 & 0.324 \\
\hline & & & & Th/U & $>4.50$ & $>0.80$ \\
\hline & & & & $\mathrm{La} / \mathrm{Y}$ & 2.5 & \\
\hline $\mathrm{Mg} / \mathrm{Mg}+\mathrm{Fe}^{2+}$ & 0.663 & 0.650 & 0.766 & $\mathrm{Ce} / \mathrm{Y}$ & 4.9 & \\
\hline$\sum \mathrm{Or}, \mathrm{Ab}, \mathrm{Ne}, \mathrm{Q}$ & 33.16 & 34.76 & 91.62 & & & \\
\hline An mol. $\%$ & 60.63 & 66.83 & 2.00 & & & \\
\hline
\end{tabular}

Major and trace element analyses (RL3A, RL3) after Forsyth (1989) ${ }^{1}$ and $(\mathrm{RL} 19)^{2}$ this paper.

sodic ( ne $_{82-83}$ ), with compositions suggesting $\mathrm{T}<500^{\circ} \mathrm{C}$, based on experimental isotherms. Clinopyroxene remained diopsidic, but became more Ti- and Fe-rich. Mg-bearing ulvospinel ( $\mathrm{usp}_{42-60}$ ) formed the distinct Fe-Ti oxide. Such assemblages crystallising from hydrous nephelinitic magma would begin at around $880-890^{\circ} \mathrm{C}$ at $0.1 \mathrm{GPa}$, based on the diopside-albite-nepheline phase relationships (Patic et al. 2000) assuming sodic anorthoclase and albite are relatively close in composition. This is higher than the $\mathrm{T}$ indicated by the nepheline compositions, but the latter may be low due to hydrous vapour exchanges.

\section{Feldspathic crystallisation}

Alkali feldspar formed a principal K-rich phase (sanidine, or $_{52-53}$ ) in these assemblages (RL6), as Si, Ti, Fe and alkalis, particularly $\mathrm{K}$, became concentrated with fractionation. Although subordinate, olivine remained magnesian $\left(\mathrm{fo}_{75}\right)$ while nepheline remained sodic $\left(\right.$ ne $_{81-83}$ ) with a composition similar to the low $\mathrm{T}\left(<500^{\circ} \mathrm{C}\right)$ nepheline in the host nephelinite. Clinopyroxene is Ti-enriched diopside and ulvospinel ( $\mathrm{usp}_{47}$ ) is the accessory oxide.
In the most evolved assemblages, K-rich alkali feldspar (or $49-52$ ) appears without nepheline in alkali syenites (RL15). Clinopyroxenes enriched in $\mathrm{Ti}, \mathrm{Fe}$ and $\mathrm{Na}$ on their rims, pass from strongly zoned diopside (fig. 5), through aegirineaugite $\left(\mathrm{acm}_{23-37}\right)$ into aegirine $\left(\mathrm{acm}_{78-83}\right)$. Amphiboles in the hydrous residues are $\mathrm{K}$ - and $\mathrm{Ti}$ - rich richterites. They are deficient in their cation sites, regardless of element and Fe valency assignments. Other elements not analysed in the EMP array may be present, as detected in certain amphibole associations elsewhere (Eggins et al. 1998, Preston et al. 2000), but such assignments remain problematic.

The feldspathic assemblages are commonly subspherical with the main phases radiating inwards from the margins, suggesting cavity infilling during final consolidation of the nephelinite. This contrasts with the irregular and vein-like mafic assemblages. These feldspathic residues resemble invariant point equilibrium assemblage compositions found for nephelinite-like compositions in the experimental diopside-albite-nepheline system at $\mathrm{P}\left(\mathrm{H}_{2} \mathrm{O}\right)=\mathrm{P}($ Total $)=$ $0.2 \mathrm{GPa}$ (Patic et al. 2000). 


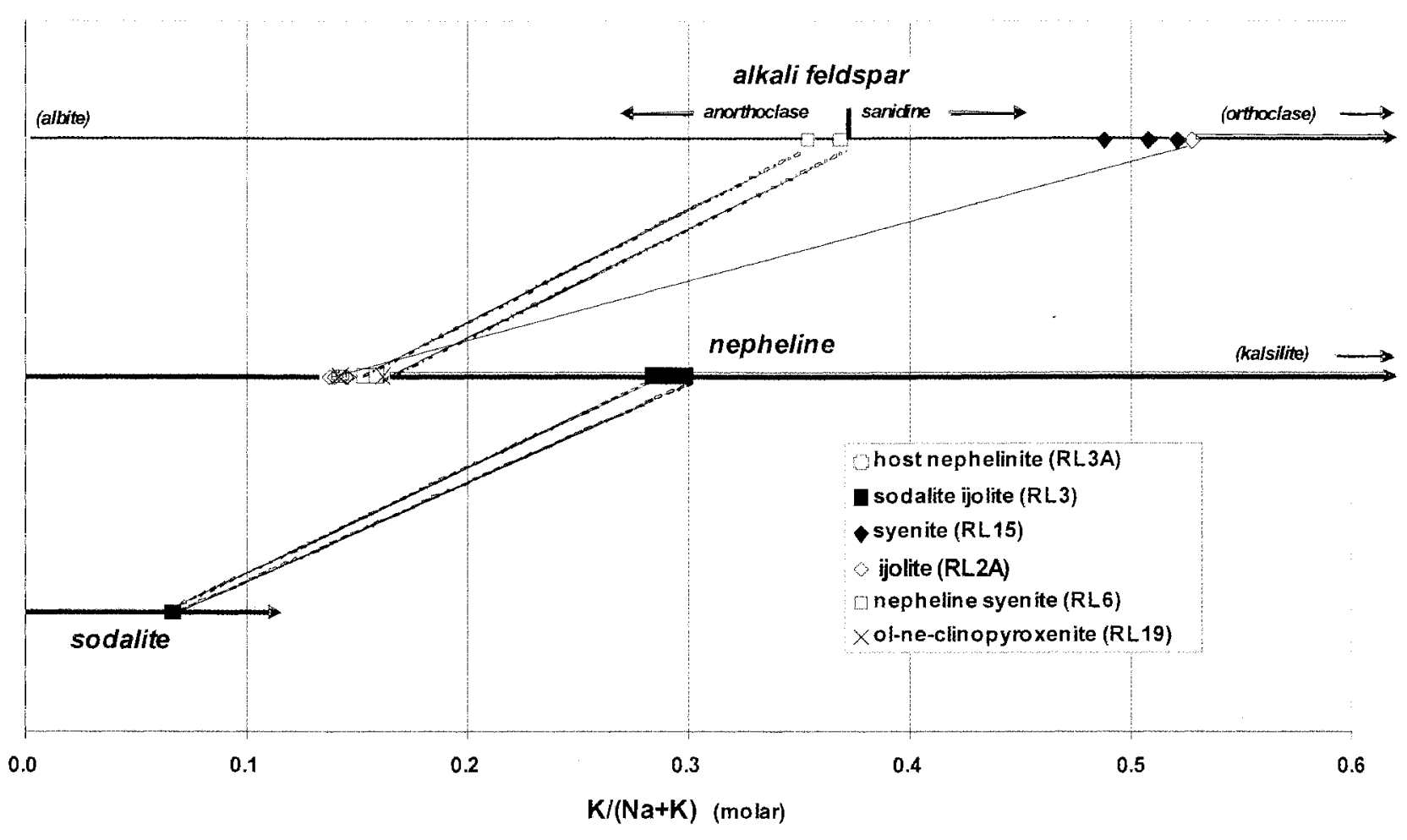

FIG. 4 - Plots of feldspathoid and feldspar compositions, from Round Lagoon nephelinite and pegmatoids: $K /(N a+K)$ molar values for alkali feldspar, nepheline and sodalite, with symbols shown in legend for different samples. The various connecting lines link co-existing phases in the same sample.

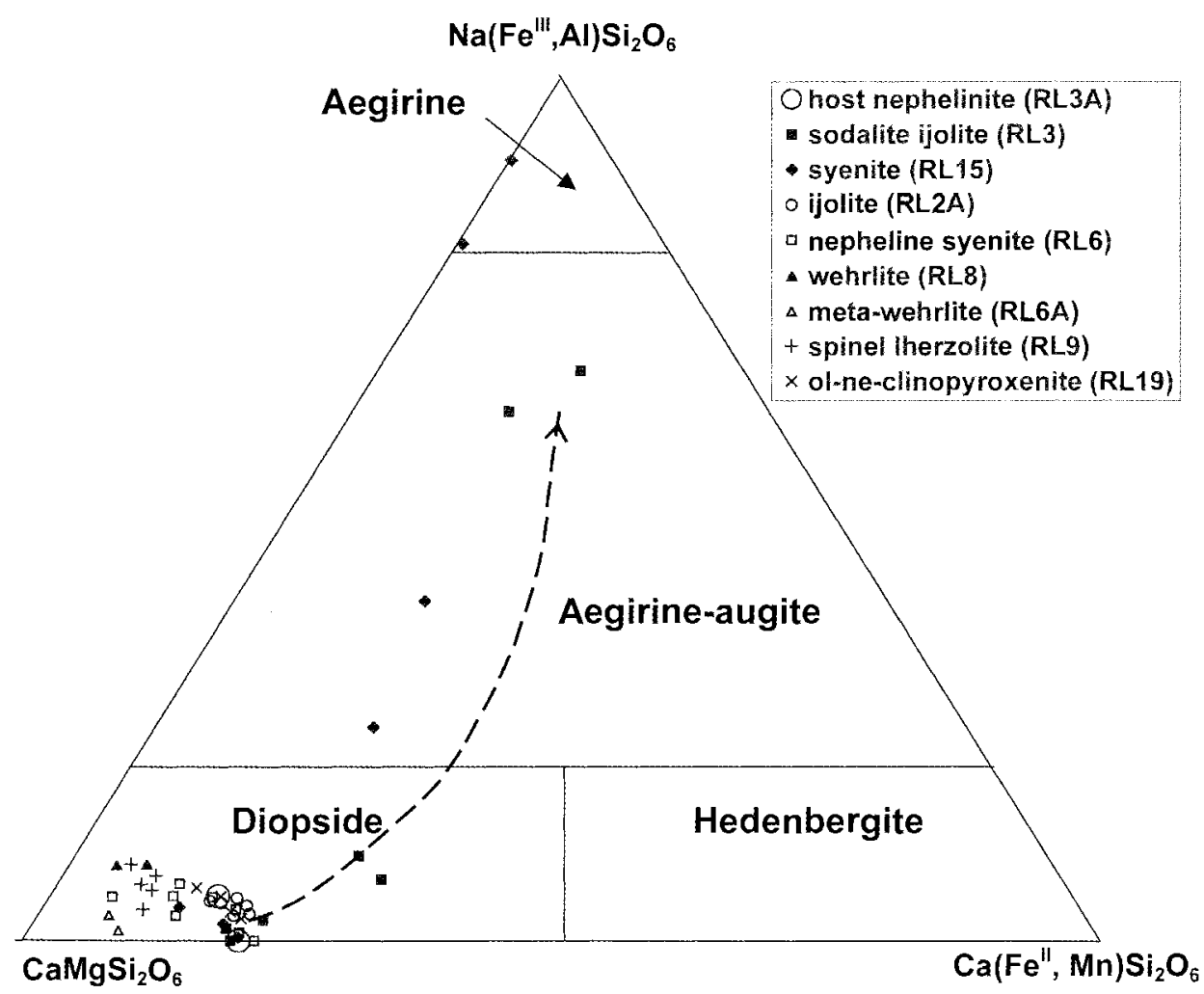

FIG. 5 - Compositional plots, Round Lagoon analysed pyroxenes, on a triangular end member Diopside - Hedenbergite Aegirine diagram, with symbols after listed samples. The trend line of increasing Na is from analyses for Inverell, NSW, nephelinite and pegmatoids shown by Wilkinson (1977). 


\section{Fractionation sequence}

The mineral paragenesis in the Round Lagoon fractionates suggests progressive, but discontinuous, crystallisation of assemblages within a cooling solidifying nephelinite magma, as follows: Wehrlites $\rightarrow$ Olivine-clinopyroxenites $\rightarrow$ Sodaliteijolites $\rightarrow$ Ijolites $\rightarrow$ Nepheline syenites $\rightarrow$ Alkali syenites. Within this series, $\mathrm{Mg}$ depletion took place in olivines ( $\mathrm{fo}_{91}$ $\left.\rightarrow \mathrm{fo}_{67}\right)$ and clinopyroxenes $\left(\mathrm{en}_{55} \rightarrow \mathrm{en}_{6}\right)$, which became more $\mathrm{Na}$ and Fe-rich $\left(\mathrm{acm}_{\mathrm{o}} \rightarrow \mathrm{acm}_{83}\right)$. Accessory oxides were spinel $\left(\mathrm{spl}_{83} \rightarrow \mathrm{spl}_{1} ; \mathrm{Chr}_{14} \rightarrow \mathrm{chr}_{1}\right.$ ) in ultramafic associations and magnetite-ulvospinel $\left(\operatorname{mag}_{1} \rightarrow \operatorname{mag}_{34} ;\right.$ usp $\left._{2} \rightarrow \mathrm{usp}_{61}\right)$ in mafic and feldspathic associations.

Bulk analyses of the host and cognate assemblages are limited in this study, but a sodalite-ijolite analysis (table 6) defines some trends in major element composition with low-pressure fractionation (fig. 2). The depletions in $\mathrm{Cr}$ and $\mathrm{Ni}$ and enrichments in incompatible elements such as $\mathrm{Ba}, \mathrm{Sr}, \mathrm{Rb}, \mathrm{Zr}$ and $\mathrm{Nb}$ reflect olivine, pyroxene and spinel crystallisation. The host and sodalite-ijolite bulk compositions do not show extensive separation related to prolonged olivine-clinopyroxene-nepheline crystallisation within the phase equilibrium nepheline-diopside-silica system (Wilkinson 1977). This may suggest rapid, nonequilibrium conditions prevailed during sodalite-ijolite crystallisation, probably initiated by a Cl-rich flux, perhaps accompanied by some magma mixing.

\section{Comparisons with other nephelinite pegmatoids}

Examples of pegmatoids in nephelinites in Tasmania, eastern Australia and basalt fields elsewhere are sparse (London 2008). Pegmatoids are also recorded in melilite-bearing or basanitic hosts as in the Shannon Tier melilitite and Table Cape teschenite (Sutherland et al. 2004). Transitional basanite nephelinites also contain coarse-grained differentiations (Wittke \& Holm 1996). These pegmatoids, however, involve further Ca-rich phases such as melilite or plagioclase in their fractionation and only simpler nephelinite fractionates are considered here.

\section{Australia}

Pegmatoids in Tasmanian nephelinites are recorded at Boat Harbour, East Arm (Tamar River) and West Scottsdale, but detailed mineral analyses are only available for the Boat Harbour pegmatoid. That pegmatoid is transitional in mode between Round Lagoon sodalite-ijolite (RL3) and nepheline-syenite (RL6). Sodalite is less potassic ( $\mathrm{k}-\mathrm{sdl}_{1}$ of $\mathrm{k}$-sdl $)$, nepheline is intermediate in $\mathrm{K}$-content $\left(\mathrm{kls}_{19} c f \mathrm{kl}_{15}\right.$ $\left.{ }_{30}\right)$ and alkali feldspar is more potassic $\left(\right.$ or $_{21} c f$ or $\left._{53-59}\right)$, while clinopyroxene has higher $\mathrm{Mg}\left(\mathrm{en}_{47} c f\right.$ en $\left.{ }_{41-42}\right)$ and olivine intermediate $\mathrm{Mg}\left(\mathrm{fo}_{72} c f \mathrm{fo}_{64-75}\right)$, compared to Round Lagoon phases. Nepheline compositions suggest similar crystallisation temperatures to those in the sodalite-ijolite pegmatoid at Round Lagoon $\left(-700^{\circ} \mathrm{C}\right)$.

Melanocratic and leucocratic pegmatoids at Inverell (Wilkinson 1977 ) respectively resemble ijolite (RL2A) and nepheline-syenite (RL6) at Round Lagoon. Inverell melanocratic variants, however, have less sodic nepheline $\left(n_{79}\right.$ of ne $\left.{ }_{83}\right)$ and more potassic alkali feldspar $\left(\right.$ or $_{57-64}$ of $\mathrm{or}_{37-52}$ ), while clinopyroxenes have lower $\mathrm{Ti}$ and $\mathrm{Al}$ and olivine has lower $\mathrm{Mg}\left(\mathrm{fo}_{60} \mathrm{cf} \mathrm{fo}_{67}\right)$. Nepheline compositions suggest formation at $-500^{\circ} \mathrm{C}$, compared to $<500^{\circ} \mathrm{C}$ at Round Lagoon.
Inverell host nephelinite is more evolved than Round Lagoon nephelinite ( $\mathrm{Mg} \# 0.63$ cf 0.66$)$, but has a similar $\mathrm{Na}_{2} \mathrm{O} / \mathrm{K}_{2} \mathrm{O}$ ratio (1.95 of 1.96 , table 6 ). The higher $\mathrm{Ca}$ and $\mathrm{Al}$ contents in the Inverell nephelinite may reflect its more olivine-dominated fractionation, but other magmatic differences may also apply.

An alkaline pegmatoidal clot found in a Victorian nephelinite differs from the Tasmanian examples in lacking nepheline and containing quartz, giving it a problematic relationship to its host (Ferguson 1977).

\section{Argentina}

A well studied nephelinite-pegmatoid exposure at La Madera, Córdoba (Galliski et al. 2004), is derived from a more primitive parental magma than for the Australian examples (Mg\#0.70 cf 0.63-0.66). The presence of both melanocratic and leucocratic pegmatoids parallels the Inverell situation more closely than the more diverse assemblages at Round Lagoon. La Madera melanocratic assemblages are closest to Round Lagoon ijolites (RL2A), but lack sodalite, alkali feldspar and olivine. La Madera nephelines are more potassic $\left(\mathrm{Ks}_{30-34}\right.$ of $\left.\mathrm{Ks}_{15-30}\right)$, while the Ti, Al, Na-bearing diopsides and Ti-rich magnetites overlap the Round Lagoon mineral compositions. The pyroxenes show $\mathrm{Na}$ and $\mathrm{Fe}^{3+}$ enrichment trends, but do not extend to aegirine as in the extreme Round Lagoon fractionates. Amphiboles have less Si and more Ti and $\mathrm{K}$ than Round Lagoon amphiboles, and are mostly katophorites rather than richterites.

Nepheline compositions in La Madera pegmatoids give more restricted $\mathrm{T}$ crystallisation ranges than Round Lagoon pegmatoids $\left(750-800^{\circ} \mathrm{C}\right.$ of $\left.<500-980^{\circ} \mathrm{C}\right)$. Other mineral phases, not recorded in Round Lagoon assemblages, include perovskite, analcime and phillipsite-Na. The leucocratic pegmatoids at La Madera are hydrous and carbonated assemblages that indicated liquid immiscibility, a process less evident in Round Lagoon fractionation. Although injected in surface extrusions, La Madera pegmatoids provide insight into subsurface fractionation at Round Lagoon.

\section{Round Lagoon low-P magmatic evolution}

The various pegmatoids in the nephelinite demonstrate a stepped fractionation process. Separation of $\mathrm{Mg}$-rich olivine, $\mathrm{Mg}$-Ca-rich clinopyroxene and $\mathrm{Mg}$-rich spinel during initial cooling from $1140^{\circ} \mathrm{C}$ depleted the magma in considerable $\mathrm{Mg}$ and some $\mathrm{Ca}$. The greater activity of $\mathrm{MgO}$ over that of other oxides expands the diopside field (Deer et al. 1997a, Gallisky et al. 2004), so that wehrlitic cumulates gave way to olivine clinopyroxenite cumulates. A change, from $\mathrm{Cr}$ - and $\mathrm{Mg}$-rich clinopyroxene to more calcic clinopyroxene, introduced two separate pyroxene components into the pegmatoid sequence. Differences in clinopyroxene phenocryst compositions related to changes in magma chamber compositions are known from phenocrysts generated below nephelinite volcanoes (e.g., at Napak volcano; Simonetti et al. 1996), but the precise causes that produced the two distinct Round Lagoon types ( $\mathrm{Cr}$ - and $\mathrm{Na}$-bearing augite and $\mathrm{Ti}$ - and $\mathrm{Al}$-bearing diopside) await more detailed study. As alkalis and Fe increased in the evolving magma and temperatures dropped below $1000^{\circ} \mathrm{C}$, nepheline became an important crystallising phase and the pyroxene composition moved towards aegerine-augite, rather than less stable hedenbergite (Galliski et al. 2004). With $\mathrm{Al}$ entering into nepheline, distinct changes in oxide crystallisation favoured ulvospinels over spinels. 
As Si contents increased, alkali feldspar joined the other crystallising phases to form ijolites and nepheline syenites. These solidified below $850^{\circ} \mathrm{C}$, as typifies lower temperature derivatives observed in the diopside-albitenepheline experimental system (Patic et al. 2000). Such assemblages crystallise from host magmas similar to Round Lagoon nephelinite after removal of about $10 \%$ olivine, 20-25\% diopside, 10-15\% nepheline, 5-6\% Ti-rich magnetite and 3-4\% apatite (Wilkinson \& Stolz 1983). These derivatives are limited in volume and are unlikely to deliver phonolitic eruptions at the surface. Furthermore, their mineral compositions are distinct from those in rare phonolites found in Tasmania (Everard et al. 2004). In those phonolites, nepheline compositions suggest higher crystallisation $\mathrm{T}$, sodalite lacks a $\mathrm{K}$-component, alkali feldspar includes two types, a Ba-rich and a high K-type, core clinopyroxenes are hedenbergite, amphiboles are arfvedsonite-eckermanite and hastingsite-pargasite, not richterite, and olivine is fayalite. This indicates a different genetic process. The chemical fractionation process within the Round Lagoon nephelinite may include elements of a gas-filter pressure process, where segregation of interstitial liquid takes place after $35-50 \%$ crystallisation of the host magma (Rogan et al. 1996).

The end fractionate in Round Lagoon nephelinite is a sanidine-diopside-amphibole assemblage, equivalent to alkaline trachyte. The overall late $\mathrm{Na}$-enrichment that developed in the clinopyroxenes relative to $\mathrm{Ca}$, i.e., $\mathrm{Na} /$ $(\mathrm{Na}+\mathrm{Ca})$ atomic ratio and $\mathrm{Mg}$, i.e., cations per formula unit, ranges from $\mathrm{Na} /(\mathrm{Na}+\mathrm{Ca}) 0.63 \rightarrow 0.91$ and $\mathrm{Mg}$ $0.46 \rightarrow 0.13$. This follows the evolved pyroxene trend for Teneriffe sodalite-nepheline syenites (Wolff 1987), but at higher Mg levels. It marks an extreme Mg-rich trend (fig. 5) within late-stage alkali pyroxene trends in fractionated alkaline magmas (see Wilkinson 1977, Wolff 1987, Deer et al. 1997b, Ridolfi et al. 2006). It lies distant from trends related to carbonatitic and more saturated alkaline magma associations.

\section{Mantle xenolith alterations}

Early studies on mantle xenolith minerals from undersaturated alkaline lavas found blebs of glassy melts in some assemblages. This led to debates on their origin, whether through a mantle process or a transport decompression process (Scribano 1986, O'Connor etal. 1996). In Australian (Victoria) mantle xenolith studies, Yaxley et al. (1997) discounted both equilibrium partial melting of peridotite and migration of exotic metasomatic agents for glass generation. They favoured disequilibrium melting of in situ metasomatic materials, either through heating or decompressive effects. In a wide study of xenoliths, Coltorti et al. (2000) precluded any secondary mineral fractionations as a mechanism for forming the glasses and were able to distinguish glasses derived from mafic melt metasomatism, either sodic or potassic, or carbonatitic metasomatism. Views on whether these glasses formed at mantle depths or by lower pressure decompression during xenolith transport have adherents on both sides. Relatively high-pressure heating, with influx of various metasomatising agents was favoured for melt pockets in xenoliths from a western Hungary basalt field (Bali et al. 2008). In contrast, glass compositions and mineral reactions in mantle peridotite were mimicked in melting experiments at one atmosphere with a synthetic alkali host composition (Shaw \& Dingwall 2008).
Round Lagoon mantle xenoliths show variable alteration in their mineralogy and textures, particularly through reaction replacements with formation of glassy melt zones. Metawehrlites $(\mathrm{pl}, 2 \mathrm{H})$ which retain deformation zoned olivine, sieve-texture diopsides and minor $\mathrm{Cr}$-bearing spinel amid extensive recrystallised glassy zones may represent spinel lherzolites, where much orthopyroxene and spinel was reacted and lost constituent elements into the recrystallising melts. The low $\mathrm{Al}$ contents and $\mathrm{Al}$ co-ordination in the sievedtextured diopsides resemble low-pressure diopside more than the diopside in pristine spinel lherzolite (table 5), suggesting decompression melting at high crustal levels.

Noticeable amounts of reaction products and glassy melt occur within Round Lagoon mantle assemblages compared with some other Tasmanian mantle xenolith suites. This may result from prolonged heating and fluid interaction of the xenoliths during their retention in a relatively slow cooling, fractionating magmatic feeder.

\section{Round Lagoon eruptive reconstruction}

The pegmatoids in this plug differ from those formed in ponded surface nephelinite lavas (e.g., at Inverell, La Madera). In the ponded situations, fractionated melts formed during fluxing in cooling horizontal bodies (Wilkinson 1977, Galliski et al. 2004). If such lava roofs formed above the Round Lagoon feeder, they have since been eroded away. The Tiers escarpment would be much farther east 25 million years ago, so that former related plateau lavas were possible above this feeder.

Fractionation in the plug operated in a vertical, upwardstreaming setting, probably concentrating in favoured zones where enriched flux components $\left(\mathrm{PO}_{4}^{3-}, \mathrm{Cl}^{1-}, \mathrm{H}_{2} \mathrm{O}, \mathrm{CO}_{2}\right)$ inhibited nucleation and increased rates of disequilibrium crystal growth. Rare examples of vertical pegmatoid dykes occur in the plug, but the pegmatoid bodies largely resemble xenoliths in shape and size and may represent fractionation zones disrupted during ongoing magma discharge. The late, less coarse, spherical infilled pegmatoids probably mark residual fractionates that formed in a largely degassed, consolidating magma after final lava discharge.

\section{ACKNOWLEDGEMENTS}

D.F. Hendry made valuable additional EMP analyses of the minerals for this study when attached to the Department of Geology and Geophysics, University of Sydney. Facilities for EMP mineral analyses were provided by the Electron Microscope Unit, University of Sydney and the School of Earth Sciences, Macquarie University. The Australian Museum Trust provided funding for the study and the Tasmanian Geological Survey and Mineral Resources Tasmania, Hobart, provided field and laboratory support. The script was read by L.M. Barron, Research Associate, Australian Museum, Sydney. Script preparation was assisted by P. Bayliss, R. Springthorpe, Australian Museum, and F. Kelly, St Peters, Sydney. Constructive reviews of the script were made by $P$. Carr, School of Earth \& Environmental Sciences, University of Wollongong and I. Roach, Department of Earth and Marine Sciences, Australian National University, Canberra. JLE and SMF publish with permission of the Director, Mineral Resources Tasmania.

This paper is dedicated to John F.G. Wilkinson, for his work on low-pressure fractionation of undersaturated alkaline lavas at the University of New England, Armidale, NSW. 


\section{REFERENCES}

Adam, J. 1990: The geochemisrry and experimental petrology of sodic alkaline basalts from Oatlands, Tasmania. Journal of Petrology 31: 1201-1223.

Adam, J., Oberti, R., Cámara, F. \& Green, T.H. 2007: An electron microprobe LAM-ICP-MS and single-crystal $\mathrm{X}$-ray structure refinement study of the effects of pressures, melt $-\mathrm{H}_{2} \mathrm{O}$ concentration and $f \mathrm{O}_{2}$ on experimentally produced basaltic amphiboles. European Journal of Mineralogy 19: 641-655.

Bali, E., Zanetti, A., Szabó, C., Peate, D.W. \& Waight, T.E. 2008: A micro-scale investigation of melt production and extraction in the upper mantle based on silicate melt pockets in ultramafic xenoliths from the Bakony-Balaton Highland volcanic field (Western Hungary). Contributions to Mineralogy and Petrology 155: 165-179.

Briggs, R.M., Rosenberg, M.D., Lange, P.J. de, Itaya, T., King, P.R. \& Price, R.C. 1997: Geology and geochemistry of Gannet (Karewa) Island, Tasman Sea: a rift-related nephelinitic tuff ring. New Zealand Journal of Geology and Geophysics 40: 263-273.

Bultitude, R.J. \& Green, D.H. 1971: Experimental study of crystal-liquid relationships at high pressures in olivine nephelinite and basanite compositions. Journal of Petrology 12: 121-147.

Cebeira, J.M. 1990: PX: A program for pyroxene classification and calculation. American Mineralogist 75: 1426-1427.

Chazot, G., Menzies, M.A. \& Harte, B. 1996: Determination of partition coefficients between apatite, clinopyroxene, amphibole and melt in natural spinel lherzolites from Yemen: implications of wet melting in the lithospheric mantle. Geochimica et Cosmochimica Acta 60: 423-427.

Coltorti, M., Beccaluva, L., Bonadiman, C., Salvini, L. \& Siena, F. 2000: Glasses in mantle xenoliths as geochemical indicators of metsomatic agents. Earth and Planetary Science Letters 183: 303-320.

Deer, W.A., Howie, R.A. \& Zussman, J. 1982: Olivine. In Rockforming Minerals, Volume 1A. Second Edition, Orthosilicates, Longman, London: 3-336.

Deer, W.A., Howie, R.A. \& Zussman, J. 1997a: DiopsideHedenbergite. Rock-forming Minerals, Volume 2B, Second Edition, Double-chain Silicates. The Geological Society, London: $764 \mathrm{pp}$.

Deer, W.A., Howie, R.A. \& Zussman, J. 1997b: Aegirine. Rockforming Minerals, Volume 2B, Second Edition, Double-chain Silicates. The Geological Society, London: 482-519.

Direen, N.G. \& Leaman, D.E. 1997: Geophysical modelling of structure and tectonostratigraphic history of the Longford Basin, northern Tasmania. Exploration Geophysics 28: 29-33.

Edgar, A.D. 1984: Chemistry, occurrence and paragenesis of feldspathoids: a review. In Brown, W.L. (ed.): Feldspars and Feldspathoids: Structures, Properties and Occurences. D. Reidal, Dorrecht, The Netherlands: 501-532.

Eggins, S.M., Rudnick, R.L. \& McDonough, W.F. 1998: The composition of peridotites and their minerals: a laserablation ICP-Ms study. Earth and Planetary Sciences Letters 154: 53-71.

Everard, J.L. 2001: Inclusions of high pressure origin in Tasmanian Cenozoic basalts: a catalogue of localities. Tasmanian Geological Survey Record 2001/09.

Everard, J.L., Sutherland F.L. \& Zwingmann, H. 2004: A Cretaceous phonolite dyke from the Tomahawk River, Northeast Tasmania. Papers and Proceedings of the Royal Society of Tasmania 138: 11-33.

Ferguson, A.K. 1977: A note on a ramsayite-bearing pegmatoidal clot in a mela-nephelinite from the Older Volcanics near Bacchus Marsh, Victoria. Journal of the Geological Society of Australia 24: 491-494.

Fodor, R.V., Bauer, G.R. \& Keil, K. 1982: Ultramafic inclusions and megacrystals in olivine nephelinite, Aitutaki Island, Cook Islands. New Zealand Journal of Geology and Geophysics 25: 67-76.

Forsyth, S.M. 1989: Geological Survey Explanatory Report. Geological Atlas 1:50 000 Series Sheet $61(8313 \mathrm{~N})$. Interlaken. Tasmanian Department of Mines.

Galliski, M.A., Lira, R. \& Dorais, M.J. 2004: Low pressure differentiation of melanephelinitic magma and the origin of ijolitic pegmatites at La Madera, Córboda, Argentina. Canadian Mineralogist 42: 1799-1823.

Green, D.H. \& Falloon, T.J. 1998: Pyrolite: A Ringwood concept and its current expression. In Jackson, I. (ed.): The Earth's Mantle. Composition, Structure and Evolution. Cambridge University Press, Cambridge: 311-378.

Hamilton, D.L. 1961: Nephelines as crystallisation temperature indicators. Journal of Geology 69: 321-329.

Hutchison, C.S. 1974: Laboratory Handbook of Petrographic Techniques. John Wiley \& Sons, New York: 527 pp.

Johnson, R.W. (ed.) 1989: Intraplate Volcanism in Eastern Australia and New Zealand. Cambridge University Press, Cambridge: 408 pp.

Kretz, R. 1983: Symbols for rock-forming minerals. American Mineralogist 68: 277-279.

Leake, B.E. \& contributers 1997: Nomenclature of amphiboles: report of the sub-committee on Amphiboles of the International Mineralogical Association, Commission of New Minerals and Mineral Names. Canadian Mineralogist 35: 219-246.

Le Maitre, R.W. (ed.) 2002: Igneous Rocks: A Classification and Glossary of Terms. Second Edition, Cambridge University Press, Cambridge: 252 pp.

London, D. 2008: Pegmatites. Mineralogical Association of Canada Special Publication 10, Quebec: 368 pp.

McDonough, W.F., McCulloch, M.J. \& Sun, S-S. 1985: Isotopic and geochemical systematics in Tertiary-Recent basalts from south eastern Australia and implications for the evaluation of the sub-continental lithosphere. Geochimica et Cosmochimicia Acta 49: 2051-2067.

Mason, D.R. 1985: Polybaric crystallisation of clinopyroxene in ankaramites of the Barrington Tops Tertiary Volcanic Field. In Sutherland, F.L., Franklin, B.J. \& Waltho, A.E. (eds): Volcanism in eastern Australia. Geological Society of Australia, New South Wales Division, Special Publication 1: 84-105.

Morimoto, N. 1988: Nomenclature of pyroxenes. Mineralogical Magazine 52: 535-550.

O'Connor, T.K., Edgar, A.D. \& Lloyd, F.E. 1996: Origin of glass in Quaternary mantle xenoliths from Meerfeldermaar, West Eifel, Germany: implications for enrichment in the lithospheric mantle. Canadian Mineralogist 34: $187-200$.

Patic, J.K., Arima, M. \& Gupta A.K. 2000: Experimental study of the system diopside-albite-nepheline at $\mathrm{P}\left(\mathrm{H}_{2} \mathrm{O}\right)=$ $P($ Total $)=2$ and $10 \mathrm{Kbar}$ and at $\mathrm{P}($ Total $)=28 \mathrm{Kbar}$. Canadian Mineralogist 38: 1177-1191.

Preston, R.J., Hole, M.J. \& Still, J. 2000: The occurrences of

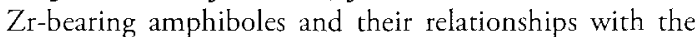
pyroxenes and biotites in the teschenite and nepheline syenites of a differentiated dolerite boss, Islay, NW Scotland. Mineralogical Magazine 64: 459-468.

Rawlinson, N., Houseman, G.A., Collins, C.D.N. \& Drummond, B.J. 2001: New evidence of Tasmania’s tectonic history from a novel seismic experiment. Geophysical Research Letters 28: 3337-3340.

Ridolfi, F., Renzulli, A., Macdonald, R. \& Upton, B.G.J. 2006: Peralkaline syenite autoliths from Kilombe volcano, Kenya Rift Valley: Evidence for subvolcanic interaction with carbonatitic fluids. Lithos 91: 373-392.

Rogan, W., Blake, S. \& Smith, I. 1996: In situ chemical fractionation in thin basaltic lava flows: examples from the Auckland volcanic field, New Zealand and a general 
physical model. Journal of Volcanology and Geothermal Research 74: 89-99.

Scribano, V. 1986: The harzburgite xenoliths in a Quaternary basanitoid lava near Scordia (Hyblean Plateau, Sicily). Rendictoni Società Italiana di Mineralogia e Petrologia 41(2): 245-255.

Shaw, C.S.J. \& Dingwell, D.B. 2008: Experimental peridotitemelt reaction at one atmosphere: a textural and chemical study. Contributions to Mineralogy and Petrology 155: 199-214.

Simonetti, A., Shore, M. \& Bell, K. 1996: Diopside phenocrysts from nephelinitic lavas, Napak volcano, eastern Uganda: Evidence for magma mixing. Canadian Mineralogist 34: $411-421$.

Smith, I.E.M., Blake, S., Wilson, C.J.N. \& Houghton, B.F. 2008: Deep seated fractionation during the rise of a small volume basalt magma batch: Crater Hill, Auckland, New Zealand. Contributions to Mineralogy and Petrology 155 : $511-527$.

Spera, F.J. 1984: Carbon dioxide in petrogenesis: 111. Role of volatiles in the ascent of alkaline magma with special reference to xenolith-bearing mafic lavas. Contributions to Mineralogy and Petrology 88: 217-232.

Sun, S-S. \& McDonough, W.F. 1989: Chemical and isotopic systematics of oceanic basalts: implications for mantle composition and processes. In Saunders, A.D. \& Norry, M.J. (eds): Magnetism in the Ocean Basins. Geological Society Special Publication No. 42: 313-345.

Sutherland, F.L. 1974: High-pressure inclusions in tholeiitic basalt and the range of lherzolite-bearing magmas in the Tasmanian volcanic province. Earth and Planetary Science Letters 24: 317-232.

Sutherland, F.L. 1989: Cainozoic volcanic rocks. In Forsyth, S.M. (Compiler): Geological Survey Explanatory Report. Geological Atlas 1:50 000 Series Sheet 61 (8313N). Interlaken. Tasmanian Department of Mines: 48-60.

Sutherland. F.L. \& Hollis, J.D. 1982: Mantle-lower crust petrology from inclusions in basaltic rocks in eastern Australia - an outline. Journal of Volcanology and Geothermal Research 14: 1-29.

Sutherland F.L. and contributors 1989: Tertiary volcanism, Terciary basaltic magmas and the Tasmanian lithosphere. In Burrett, C.F. \& Martin, E.L. (eds): Geology and Mineral Resources of Tasmania. Geological Society of Australia Special Publication 15: 383-398.

Sutherland, F.L., Graham, I.T., Everard, J.L., Forsyth, S.M. \& Zwingmann, H. 2004: Cenozoic basalts, Tasmania: Landscapes, exposures, ages, petrology, geochemistry, entrainments and petrogenesis. Geological Society of Australia Field Guide A5, Sydney: $58 \mathrm{pp}$.

Sutherland, F.L., Hendry, D.F., Barron, B.J., Matthews, W.L. \& Hollis, J.D. 1996: An unusual Tasmanian Tertiary basalt sequence near Boat Harbour, Northwest Tasmania. Records of the Australian Museum 48: 131-161.
Sutherland, F.L., Raynor, L.R. \& Pogson, R.E. 2005: Table Cape xenolithic suite, northwest Tasmania: Mineralogy and implications for crust-mantle lithology and Miocene geotherms in Tasmania. Papers and Proceedings of the Royal Society of Tasmania 139: 7-22.

Ulrych, J., Lloyd, F.E., Balogh, K., Hegner, E., Langrová, A., Lang, M., Novák, J.K. \& Řanda, Z. 2005: Petrogenesis of alkali pyroxenite and ijolite xenoliths from the Tertiary Loučná-Oberweisenthal Volcanic Centre, Bohemian Massif in the light of new mineralogical, geochemical, and isotopic data. Neues Jabrbuch für Mineralogie-Abhandlungen 182: $57-59$

Ulrych, J., Pivec, E., Lang, M. \& Lloyd, F.E. 2000: Ijolite segregations in melilite nephelinite of Podhorni vrch volcano, Western Bohemia. Neues Jabrbuch für MineralogieAbhandlungen 175: 317-348.

Wass, S.Y. 1979: Multiple origins of clinopyroxenes in alkali basaltic rocks. Lithos 12: 115-132.

Wells, P.R.A. 1977: Pyroxene thermometry in simple and complex systems. Contributions to Mineralogy and Petrology 62: 129-130.

Wilkinson, J.F.G. 1977: Petrogenetic aspects of some alkali volcanic rocks. Journal \& Proceedings of the Royal Society of New South Wales 110: 117-138.

Wilkinson, J.F.G. \& Stolz, A.J. 1983: Low pressure-fractionation of strongly undersaturated alkaline ultrabasic magma: the olivine-melilite-nephelinite at Moiliili, Oahu, Hawaii. Contributions to Mineralogy and Petrology 83: 363-374.

Wittke, J.H. \& Holm, R.F. 1996: The association basanitic nephelinite-feldspar ijolite-nepheline monzosyenite at House Mountain volcano, north-central Arizona. Canadian Mineralogist 34: 221-240.

Wolff, J.A. 1987: Crystallisation of nepheline syenite in a subvolcanic magma system: Teneriffe, Canary Islands. Lithos 20: 207-223.

Woodland, A.B. \& Jugo, P.J. 2007: A complex magmatic system beneath the Devès volcanic field, Massif Central, France: evidence from clinopyroxene megacrysts. Contributions to Mineralogy and Petrology 153: 719-731.

Yavuz, F. 1996: Amphcal: A quick basic program for determining the amphibole name from electron microprobe analyses using the IMA rules. Computers and Geosciences 22: $101-107$.

Yaxley, G.M., Kamenestky, V., Gran, D.H. \& Falloon, T.J. 1997. Glasses in mantle xenoliths from western Victoria, Australia and their relevance to mantle processes. Earth and Planetary Science Letters 148: 433-466.

Zhou, J. \& Li, X. 2006: GeoPlot: An Excel VBA program for geochemical data plotting. Computers and Geosciences 32: $554-560$.

(accepted 7 October 2008) 